\title{
CONVERGENCE IN VARIATION AND RELATED TOPICS $\dagger$
}

\author{
BY \\ ANTHONY P. MORSE
}

1. Introduction. In recent papers $\ddagger$ by Adams and Clarkson and by Adams and Lewy the notions of convergence in variation and convergence in length have been examined. In AC it has been shown that if a sequence converges in variation and satisfies certain further restrictions which are clearly needed, the sequence of reciprocals converges in variation. The central purpose of the present paper is to determine so far as we are able the transformations which when applied to sequences of functions, preserve various types of convergence, such as convergence in variation or length and other types which we shall introduce. This paper also leads us to certain generalizations of results in $\mathrm{AC}$ and $\mathrm{AL}$, such as Theorem 5.4 wherein convergence in length is seen to be invariant under addition and multiplication when only one of the limit functions is absolutely continuous.

In $\$ 2$ we assemble certain preliminary definitions, notations, and conventions. $\$ 3$ is devoted to preliminary theorems and lemmas, among them being Theorems 3.1 and 3.2 which might be of interest in themselves, their full power, in fact, not being used in this paper. Theorem 3.2 is a substitution theorem for Lebesgue integrals which is more general than other theorems of this type known to us in literature. Certain results in $\$ 3$ are, however, obvious analogues of results in AC. Transforms of sequences are discussed in $\$ 4$, wherein Theorems 4.1 and 4.2 form the kernel of the paper. The remainder of the paper consists largely of various applications of these two theorems, convergence in length being discussed in $\$ 5$ together with convergence almost in the mean, uniform convergence in length in $\$ 6$, and strong convergence in \$7. Certain miscellaneous applications are made in $\$ 8$; these include Theorem 8.1 which points out a necessary and sufficient condition for convergence in the mean, and Theorems 8.3 and 8.4 which are generalizations of a theorem of Plessner.

2. Notation; preliminary definitions and conventions. In this paper we shall consistently use $x, y, t$ to denote real numbers or variables, and use the

$\dagger$ Presented to the Society, October 31, 1936; received by the editors May 22, 1936.

$\ddagger$ Adams and Clarkson, On convergence in variation, Bulletin of the American Mathematical Society, vol. 40 (1934), pp. 413-417. Adams and Lewy, On convergence in length, Duke Mathematical Journal, vol. 1 (1935); pp. 19-26. Hereinafter these papers will be referred to as AC and AL, respectively. 
letters $X, Y, \xi, \psi, \Psi, \eta, u, v, U, V$ with or without subscripts to denote realvalued functions. All other functions are to be regarded as complex functions of a real variable unless the contrary is expressly stated. We shall also employ $a$ and $b$ with or without subscripts to designate real numbers with $a<b$.

If $Q$ is a condition involving $x$, then $\underset{x}{E}[Q]$ is a set defined as follows: A point $x$ belongs to $\underset{x}{E}[Q]$ if $x$ satisfies the condition $Q$. We use the notation $\left[x_{1}, x_{2}\right]$ to denote a closed interval.

If $f$ is a function and if $[a, b]$ is included in its domain, then the symbol $T_{a}{ }^{b}(f)$ (read the total variation from $a$ to $b$ of $f$ ) will be used to denote the least upper bound (finite or infinite) of numbers of the form

$$
\sum_{j=1}^{k}\left|f\left(t_{j}\right)-f\left(t_{i-1}\right)\right|
$$

where $a=t_{0}<t_{1}<t_{2}<\cdots<t_{k}=b$. We define $T_{b}{ }^{a}(f)=-T_{a}{ }^{b}(f)$ and $T_{a}{ }^{a}(f)=0$. If $T_{a}{ }^{b}(f)<\infty$, then $f$ is said to be of b.v. (bounded variation) on $[a, b]$. Since it will sometimes be necessary to display the variable with respect to which the total variation is taken, we employ the notation $T_{t=a}^{b} f(t)$ as an alternate for $T_{a}{ }^{b}(f)$.

We shall use a.c. as an abbreviation for absolute continuity and employ p.p. to denote almost everywhere (presque partout), and designate the outer measure of a set $R$ by $|R|$. It will also be convenient to refer to Euclidean space of $n$ dimensions as simply $n$-space. Furthermore, a function will be said to be increasing on a set if it is strictly increasing there, a function will be said to be monotone on a set if it is either non-increasing or non-decreasing there.

The following convention, will be used throughout the paper. If $f$ is defined on $[a, b]$, then the function $f^{\prime}$ is defined on $[a, b]$ by the following relations:

$f^{\prime}(t)=$ the derivative of $f$ at $t$ wherever it exists finite,

$f^{\prime}(t)=0$ for all other $t$ on $[a, b]$.

We also agree: If $[a, b]$ is the domain of $f$ and if $\lim _{h \rightarrow 0+} f(t+h)$ exists for $a \leqq t<b$ and if $\lim _{h \rightarrow 0+} f(t-h)$ exists for $a<t \leqq b$, then $f(t+)$ and $f(t-)$ are defined for $t$ on $[a, b]$ as follows:

$$
\begin{aligned}
f(a-)=f(a) ; \quad f(b+)=f(b) ; \quad f(t+) & =\lim _{h \rightarrow 0+} f(t+h), \quad a \leqq t<b ; \\
f(t-) & =\lim _{h \rightarrow 0+} f(t-h), \quad a<t \leqq b .
\end{aligned}
$$

We shall denote by $C R$ the set of all finite-valued complex functions whose domain is $[0,1]$. If $f$ and $g$ are any points in $C R$ and if $\alpha$ is any complex number, then by $f+g$ is meant that point $G$ in $C R$ such that $G(t)=f(t)+g(t)$ for $t$ on $[0,1]$, by $f \cdot g$ is meant that point $G$ in $C R$ such that $G(t)=f(t) \cdot g(t)$ for $t$ on 
$[0,1]$, by $\alpha f$ is meant that point $G$ in $C R$ such that $G(t)=(\alpha) \cdot f(t)$ for $t$ on $[0,1]$; and, provided $f$ does not vanish on $[0,1]$, by $\alpha / f$ is meant that point $G$ in $C R$ such that $G(t)=\alpha / f(t)$ for $t$ on $[0,1]$. We denote by $I$ and $\theta$ the elements of $C R$ defined respectively by

$$
I(t)=t, \quad \theta(t)=0 ; \quad 0 \leqq t \leqq 1 .
$$

If $f$ is in $C R$, then $\|f\|$, read norm of $f$, is defined as $|f(0)|+T_{0}^{1}(f)$. The space $B V$ is a subspace of $C R$ defined by $B V=(C R) \cdot E[\|f\|<\infty]$ and the subspace $R B V$ is defined by $R B V=(B V) \cdot \underset{f}{E}[f$ is real $]$.

Totally distinct from these spaces and used merely for convenience is the space $C C$ defined as the space of continuous functions on the finite complex plane to the finite complex plane.

In concluding this section we lay down the following definitions.

Definition 2.1. If $f$ is a point in $C R$ and $\phi$ is a function whose domain includes the range of $f$ and whose range is included in the set of finite complex numbers (finite complex plane), then $\phi: f$ is defined to be that point $G$ in $C R$ for which $G(t)=\phi\{f(t)\}, 0 \leqq t \leqq 1$.

Definition 2.2. If $Y$ is a real point in $C R$ and $u$ is a function on a part of two-space to one-space and if $u(t, Y(t))$ is defined for $t$ on $[0,1]$, then by $(u \mid Y)$ is meant the real point $\Psi$ in $C R$ such that $\Psi(t)=u(t, Y(t)), 0 \leqq t \leqq 1$.

Definition 2.3. Convergence in variation. By $f_{n}-v \rightarrow f_{0}$, read $f_{n}$ converges in variation to $f_{0}$, is meant this: $f_{n}$ is in $B V$ for $n=0,1,2, \cdots ; f_{n}(t) \rightarrow f_{0}(t)$ for $t$ on $[0,1] ;\left\|f_{n}\right\| \rightarrow\left\|f_{0}\right\|$.

DEFINITION 2.4. Uniform convergence in variation. By $f_{n}-u v \rightarrow f_{0}$ is meant this: $f_{n}-v \rightarrow f_{0}$ with $f_{n}(t) \rightarrow f_{0}(t)$ uniformly for $t$ on $[0,1]$.

Definition 2.5. Convergence in length. By $Y_{n}-l \rightarrow Y_{0}$ is meant this: $Y_{n}$ is in $R B V$ for $n=0,1,2, \cdots ;\left(I+i Y_{n}\right)-v \rightarrow\left(I+i Y_{0}\right)$.

DeFINITION 2.6. Uniform convergence in length. By $Y_{n}-u l \rightarrow Y$ is meant this: $Y_{n}-l \rightarrow Y_{0} ; Y_{n}(t) \rightarrow Y_{0}(t)$ uniformly for $t$ on $[0,1]$.

Definition 2.7. Strong convergence. By $f_{n}-s \rightarrow f_{0}$ is meant this: $f_{n}$ is in $B V$ for $n=0,1,2, \cdots ;\left\|f_{n}-f_{0}\right\| \rightarrow 0$.

Definition 2.8. If $\alpha$ is a point in $n_{1}$-space with $\alpha=\left(\alpha_{1}, \alpha_{2}, \cdots, \alpha_{n_{1}}\right)$ and $\beta$ a point in $n_{2}$-space with $\beta=\left(\beta_{1}, \beta_{2}, \cdots, \beta_{n_{2}}\right)$, then $\alpha \circ \beta$ is the point $\left(\alpha_{1}, \alpha_{2}, \cdots, \alpha_{n_{1}}, \beta_{1}, \beta_{2}, \cdots, \beta_{n_{2}}\right)$ in $\left(n_{1}+n_{2}\right)$-space.

3. Preliminary results. In this section certain preliminary results will be actually proved while others which are quite simple or well known will simply be stated both for completeness and for use later.

From the definition of total variation it is clear that if $a \leqq c \leqq b$ with $f$ defined on $[a, b]$, then $T_{a}^{b}(f)=T_{a}{ }^{c}(f)+T_{c}^{b}(f)$. Another corollary is the fol- 
lowing semi-continuity property: the relation $f_{n}(t) \rightarrow f_{0}(t)$ for $t$ on $[a, b]$ implies $\lim \inf _{n \rightarrow \infty} T_{a}^{b}\left(f_{n}\right) \geqq T_{a}^{b}\left(f_{0}\right)$. It is likewise easily verified that the relation $f_{n}-v \rightarrow f_{0}$ with $f_{0}$ continuous implies $f_{n}-u v \rightarrow f_{0}$ (see AC, Theorem 2 and corollary to Theorem 4 ).

Of considerable use is the following

LEMMA 3.1. The relations $f_{n}-v \rightarrow f_{0}$ and $f_{n}-u v \rightarrow f_{0}$ imply respectively the relations $\dagger S_{n}(t) \rightarrow S_{0}(t)$ for $t$ on $[0,1] ; S_{n}(t) \rightarrow S_{0}(t)$, uniformly for $t$ on $[0,1]$ where $S_{n}(t)=T_{0}{ }^{t}\left(f_{n}\right)$ for $t$ on $[0,1]$.

Several properties of the norm in $C R$ are now set forth in the following lemma.

LEMMA 3.2. If $f$ and $g$ are in $C R$ and $\alpha$ is a complex number, then

$$
\|f+g\| \leqq\|f\|+\|g\|, \quad\|\alpha f\|=|\alpha| \cdot\|f\|, \quad\|f g\| \leqq\|f\| \cdot\|g\| .
$$

The first two relations are quite simple. We sketch a proof of the last which is seen to reduce to proving

$$
\left(\sum_{n=0}^{N}\left|a_{n}-a_{n-1}\right|\right) \cdot\left(\sum_{n=0}^{N}\left|b_{n}-b_{n-1}\right|\right) \geqq \sum_{n=0}^{N}\left|a_{n} b_{n}-a_{n-1} b_{n-1}\right|,
$$

where $a_{n}$ and $b_{n}$ are complex numbers $(n=0,1, \cdots, N)$ and $\left|a_{-1}\right|+\left|b_{-1}\right|=0$. Suppose the above relation, which is obviously true if $N$ is replaced by 0 , to be true for $N$ replaced by an integer $k$ with $0 \leqq k<N$. Then it follows that

$$
\begin{aligned}
& \left(\left|a_{k+1}-a_{k}\right|+\sum_{n=0}^{k}\left|a_{n}-a_{n-1}\right|\right) \cdot\left(\left|b_{k+1}-b_{k}\right|+\sum_{n=0}^{k}\left|b_{n}-b_{n-1}\right|\right) \\
& =\left|a_{k+1}-a_{k}\right| \cdot\left|b_{k+1}-b_{k}\right|+\left|a_{k+1}-a_{k}\right| \cdot \sum_{n=0}^{k}\left|b_{n}-b_{n-1}\right| \\
& \quad+\left|b_{k+1}-b_{k}\right| \cdot \sum_{n=0}^{k}\left|a_{n}-a_{n-1}\right|+\left(\sum_{n=0}^{k}\left|a_{n}-a_{n-1}\right|\right) \cdot\left(\sum_{n=0}^{k}\left|b_{n}-b_{n-1}\right|\right) \\
& \geqq\left|\left(a_{k+1}-a_{k}\right)\left(b_{k+1}-b_{k}\right)+\left(a_{k+1}-a_{k}\right) b_{k}+\left(b_{k+1}-b_{k}\right) a_{k}\right| \\
& \quad+\sum_{n=0}^{k}\left|a_{n} b_{n}-a_{n-1} b_{n-1}\right|=\sum_{n=0}^{k+1}\left|a_{n} b_{n}-a_{n-1} b_{n-1}\right| .
\end{aligned}
$$

This induction completes the proof.

The following two lemmas are particular cases of Minkowski's inequality.

† AC, corollaries to Theorems 2 and 5 . The proof given there holds equally well for the functions considered here. 
LEMMA 3.3. If $0 \leqq a_{j} \leqq b_{i}($ for $j=1,2, \cdots, k)$, then

$$
\sum_{j=1}^{k}\left(b_{j}{ }^{2}-a_{j}{ }^{2}\right)^{1 / 2} \leqq\left[\left(\sum_{j=1}^{k} b_{j}\right)^{2}-\left(\sum_{j=1}^{k} a_{j}\right)^{2}\right]^{1 / 2} .
$$

LEMMA 3.4. If $X$ and $Y$ are summable on $[a, b]$, then

$$
\begin{aligned}
\int_{a}^{b}\left\{|X(t)|+\left|Y(t)^{\circ}\right|\right\} d t \geqq \int_{a}^{b}\left(\{X(t)\}^{2}+\{Y(t)\}^{2}\right)^{1 / 2} d t \\
\geqq\left\{\left(\int_{a}^{b}|X(t)| d t\right)^{2}+\left(\int_{a}^{b}|Y(t)| d t\right)^{2}\right\}^{1 / 2} .
\end{aligned}
$$

LEMMA 3.5. If $X$ is a function defined on $[a, b]$, then $X^{\prime}$ is measurable on $[a, b]$.

This is a corollary of a theorem found in Saks, Théorie de l'Intégrale (Chapter 3, p. 47, Theorem 1).

LEMMA 3.6. Let $[a, b]$ be the domain of the function $X$ and denote $\underset{t}{E}\left[\left|X^{\prime}(t)\right|>0\right]$ by $P$. If $D$ is a set of measure 0 , then the set $P \cdot \underset{t}{E}[X(t) \varepsilon D]$ is likewise of measure 0.

Let $Q=\underset{t}{E}\left[X(t)_{\varepsilon} D\right]$ and suppose $|P Q|>0$. Define $P_{1}=\underset{t}{E}\left[X^{\prime}(t)>0\right]$. There is no loss of generality in assuming $\left|P_{1} Q\right|>0 . P_{1}$ being measurable, it is easily established that there exist positive numbers $\epsilon_{1}, \epsilon_{2}$, and a closed set $C$ such that

$$
C \subset P_{1}, \quad\left|P_{1}-C\right|<\left|P_{1} Q\right|, \quad \frac{X\left(t_{2}\right)-X\left(t_{1}\right)}{t_{2}-t_{1}} \geqq \epsilon_{1}
$$

if $t_{1} \varepsilon C, t_{2} \varepsilon C$, and $0<\left|t_{2}-t_{1}\right|<\epsilon_{2}$. Thus $|C Q|>0$, so that there exists an interval $\left[a_{0}, b_{0}\right]$ of length $<\epsilon_{2}$ for which $\left|C Q\left[a_{0}, b_{0}\right]\right|>0$. Defining $C^{*}=C \cdot\left[a_{0}, b_{0}\right]$ and $Q^{*}=C^{*} \cdot Q$ we observe $\left|Q^{*}\right|>0$ and $C^{*}$ is closed, so that if $X_{1}$ is defined on $C^{*}$ so as to coincide with $X$ and defined on the remainder of $\left[a_{0}, b_{0}\right]$, which is made up of a set of non-overlapping intervals, by linear interpolation then it appears that $X_{1}$ is continuous and increasing on $\left[a_{0}, b_{0}\right]$ with

$$
\left|X_{1}\left(t_{2}\right)-X\left(t_{1}\right)\right| \geqq \epsilon_{1}\left|t_{2}-t_{1}\right|,\left(a_{0} \leqq t_{1}, t_{2} \leqq b_{0}\right) ; X_{1}(t)=X(t),\left(t_{\varepsilon} C^{*}\right) .
$$

The continuity of $X_{1}$ follows from the existence of the derivative of $X$ at all points of $C^{*}$. Let $\tau$ be defined on the interval $\left[X_{1}\left(a_{0}\right), X_{1}\left(b_{0}\right)\right]$ by the relation $\tau\left\{X_{1}(t)\right\}=t\left(a_{0} \leqq t \leqq b_{0}\right)$. Clearly $\tau$ satisfies a Lipschitz condition on $\left[X_{1}\left(a_{0}\right), X_{1}\left(b_{0}\right)\right]$, and thus transforms the set $D \cdot\left[X_{1}\left(a_{0}\right), X_{1}\left(b_{0}\right)\right]$ which is of measure 0 , into a set $D^{*}$ of measure 0 . But

$$
Q^{*}=C^{*} Q=C^{*} \underset{t}{E}\left[X(t)_{\varepsilon} D\right]=C^{*}{ }_{t}\left[X_{1}(t)_{\varepsilon} D\right]=C^{*} D^{*} .
$$

Hence the contradiction $\left|Q^{*}\right|=0$. 
Corollary 3.1. Let $[a, b]$ be the domain of $X$ and let $[\alpha, \beta]$ include its range. If $\psi_{1}$ and $\psi_{2}$ are finite-valued functions defined on $[\alpha, \beta]$ and equal p.p. there, then for almost all $t$ on $[a, b], \psi_{1}\{X(t)\} X^{\prime}(t)=\psi_{2}\{X(t)\} X^{\prime}(t)$.

Theorem 3.1. Let $[a, b]$ be the domain of the function $X ;$ let $[\alpha, \beta]$ include its range; let $\Psi$ be a.c. on $[\alpha, \beta]$ and denote $\Psi\{X(t)\}$ by $G(t)$ for $t$ on $[a, b]$. If $X$ has a derivative p.p. on $[a, b]$, then

$$
G^{\prime}(t)=\Psi^{\prime}\{X(t)\} X^{\prime}(t)
$$

for almost all t on $[a, b]$.

Let it first be noted that the theorem does not answer the question as to whether or not $G$ is differentiable p.p. $\dagger$ We now proceed with the proof of the theorem.

Let $R=\underset{t}{E}\left[X^{\prime}(t)=0\right]$. If $|R|=0$ the next paragraph by itself furnishes a proof. However, in case $|R|>0$, it may be noted first that corresponding to $\epsilon(>0)$ there exist positive numbers $M, \epsilon_{1}$ and a closed subset $C$, of $R$, such that $|R-C|<\epsilon$ with $\left|X\left(t_{2}\right)-X\left(t_{1}\right)\right| \leqq M\left|t_{2}-t_{1}\right|$ if $t_{1} \varepsilon C, t_{2} \varepsilon C$, and $\left|t_{2}-t_{1}\right|<\epsilon_{1}$. Likewise readily verified is the existence of a function $X_{1}$ satisfying a Lipschitz condition on $[a, b]$ (the constant involved may be $>M$ ), and in addition fulfilling: $X_{1}(t)=X(t)$ for $t_{\varepsilon} C$. Now $X_{1}$ and $X$ both transform $C$ into a measurable (closed) set $C^{\prime}$ with

$$
\left|C^{\prime}\right| \leqq \int_{C}\left|X_{1}^{\prime}(t)\right| d t=\int_{C}\left|X^{\prime}(t)\right| d t=0 .
$$

But $\Psi$ being a.c. on $[\alpha, \beta]$, transforms $C^{\prime}$ into a set $C^{\prime \prime}$ likewise of measure 0 . Hence $G$ transforms $C$ into $C^{\prime \prime}$, a set of measure 0 , so that $G^{\prime}(t)=0$ for almost all $t$ in $C$; for supposing the contrary leads immediately to a contradiction of Lemma 3.6. Hence, since $\epsilon$ was arbitrary, we conclude $G^{\prime}(t)=0$ for almost all $t$ in $R$. Thus $G^{\prime}(t)=\Psi^{\prime}\{X(t)\} X^{\prime}(t)$ for almost all $t$ in $R$.

However, upon denoting $\underset{t}{E}\left[\left|X^{\prime}(t)\right|>0\right]$ by $P$, it is seen from Lemma 3.6 that

$$
\lim _{h \rightarrow 0} \frac{\Psi\{X(t+h)\}-\Psi\{X(t)\}}{X(t+h)-X(t)}=\Psi^{\prime}\{X(t)\}
$$

† In fact, from the work of N. Bary, Mathematische Annalen, vol. 103 (1930), p. 611, a definite answer to this question can be given. Let $F$ be a continuous function nowhere differentiable on $[0,1]$. There then exist functions $G_{1}, G_{2}, G_{3}$ and a.c. functions $\Psi_{1}, \Psi_{2}, \Psi_{3}, \phi_{1}, \phi_{2}, \phi_{3}$ such that

$$
F(t)=G_{1}(t)+G_{2}(t)+G_{3}(t), \quad G_{j}(t)=\Psi_{j}\left\{\phi_{j}(t)\right\}(j=1,2,3 ; 0 \leqq t \leqq 1) .
$$

Thus at least one of the functions $G_{1}, G_{2}, G_{3}$ fails to have a derivative on a set of measure $>0$ 
for almost all $t$ in $P$. Hence $G^{\prime}(t)=\Psi^{\prime}\{X(t)\} X^{\prime}(t)$ for almost all $t$ on $[a, b]$. $\dagger$

Corollary 3.2. Let $[a, b]$ be the domain of $X$ whereon it is a.c. and let $[\alpha, \beta]$ include its range. If $\Psi$ satisfies a Lipschitz condition on $[\alpha, \beta]$ or if $\Psi$ is a.c. on $[\alpha, \beta]$ with $X$ monotone on $[a, b]$, then the function $G$ defined by $G(t)=\Psi\{X(t)\}(a \leqq t \leqq b)$, is a.c. on $[a, b]$ with $G^{\prime}(t)=\Psi^{\prime}\{X(t)\} X^{\prime}(t)$ for almost all $t$ on $[a, b]$.

Corollary 3.3. Let $[a, b]$ be the domain of $X$; let $[\alpha, \beta]$ include its range; let $\Psi$ be a.c. on $[\alpha, \beta]$; and denote $\Psi\{X(t)\}$ by $G(t)$ for $t$ on $[a, b]$. If $X$ has $a$ vanishing derivative p.p. on $[a, b]$, then a necessary and sufficient condition that $G$ is a.c. on $[a, b]$ is that $\Psi$ is constant on the range of $X$.

\section{Now combining Theorem 3.1 with Corollary 3.1 we obtain}

Theorem 3.2. Let $X$ be defined on $[a, b]$ and differentiable p.p. there; let $[\alpha, \beta]$ include the range of $X$; let $\psi$ be defined, finite-valued, and summable on $[\alpha, \beta]$ and denote $\int_{\alpha}^{x} \psi(s) d s$ by $\Psi(x)$ for $x$ on $[\alpha, \beta] ;$ finally let $G(t)=\Psi\{X(t)\}$ for $t$ on $[a, b]$. Under these circumstances

$$
\int_{X(a)}^{X(t)} \psi(x) d x=\int_{a}^{t} \psi\{X(s)\} X^{\prime}(s) d s \quad(a \leqq t \leqq b)
$$

if and only if $G$ is a.c. on $[a, b]$.

LEMMA 3.7. If $f$ is defined on $[a, b]$ then

$$
T_{a}^{b}(f) \geqq \int_{a}^{b}\left|f^{\prime}(t)\right| d t
$$

the sign of equality holding if $f$ is a.c. on $[a, b]$.

$\dagger$ A slight modification in proof establishes:

If $\Psi$ satisfies Lusin's condition $N$ on $[\alpha, \beta]$ (i.e., transforms sets of measure 0 into sets of measure 0 ) and if $X$ is continuous on $[a, b]$ in addition to being differentiable p.p. there, then $G^{\prime}(t)=\Psi^{\prime}\{X(t)\} X^{\prime}(t)$ for almost all $t$ on $[a, b]$.

It may also be noted that a slight simplification in proof may be achieved by use of a theorem of N. Bary, loc. cit., p. 190.

$\ddagger$ This theorem is a generalization of results previously obtained by de la Vallee Poussin, these Transactions, vol. 16 (1915), p. 466, and by Fichtenholz, Bulletin de l'Académie Royale de Belgique, Classe des Sciences, ser. 5, vol. 8 (1922), p. 441. In the notation of the present theorem it was shown by de la Vallee Poussin that absolute continuity of $X$ implies the equivalence of the following three statements:

(i) $\int_{X(a)}^{X(b)} \psi(x) d x=\int_{a}^{b} \psi\{X(s)\} X^{\prime}(s) d s$;

(ii) $G$ is a.c.;

(iii) $\int_{a}^{b} \psi\{X(s)\} X^{\prime}(s) d s$ exists.

On the other hand Fichtenholz showed that absolute continuity of $G$ together with monotonicity and continuity of $X$ implies (i). 
LEMMA 3.8. Let $\sigma$ be monotone and continuous on $[a, b]$. If $f$ is defined on $[\sigma(a), \sigma(b)]$, then

$$
T_{t=a}^{b} f\{\sigma(t)\}=\left|T_{\sigma(a)}^{\sigma(b)}(f)\right| .
$$

LEMMA 3.9. If $f$ is a.c. on $[a, b]$ with $f^{\prime}(t)=\mu$ (constant) for almost all $t$ on $[a, b]$, then

$$
\int_{a}^{b}\left|f^{\prime}(t)-\Delta \delta^{-1}\right| d t \leqq\left(\mu^{2} \delta^{2}-|\Delta|^{2}\right)^{1 / 2}+2(\mu \delta-|\Delta|),
$$

where $\delta=b-a$ and $\Delta=f(b)-f(a)$.

The lemma is clearly true in case $\Delta=0$. In the alternative case we define

$$
F(t)=|\Delta|(f(t)-f(a)) \Delta^{-1}=\xi(t)+i \eta(t)
$$

Now (Lemma 3.4)

$$
\begin{aligned}
\mu \delta & =\int_{a}^{b}\left|f^{\prime}(t)\right| d t=\int_{a}^{b}\left|F^{\prime}(t)\right| d t=\int_{a}^{b}\left(\left\{\xi^{\prime}(t)\right\}^{2}+\left\{\eta^{\prime}(t)\right\}^{2}\right)^{1 / 2} d t \\
& \geqq\left(\left\{\int_{a}^{b}\left|\xi^{\prime}(t)\right| d t\right\}^{2}+\left\{\int_{a}^{b}\left|\eta^{\prime}(t)\right| d t\right\}^{2}\right)^{1 / 2} \\
& \geqq\left(\left\{\int_{a}^{b} \xi^{\prime}(t) d t\right\}^{2}+\left\{\int_{a}^{b}\left|\eta^{\prime}(t)\right| d t\right\}^{2}\right)^{1 / 2} \\
& =\left(|\Delta|^{2}-\left\{\int_{a}^{b}\left|\eta^{\prime}(t)\right| d t\right\}^{2}\right)^{1 / 2}
\end{aligned}
$$

whence $\left(\mu^{2} \delta^{2}-|\Delta|^{2}\right) \geqq\left\{\int_{a}^{b}\left|\eta^{\prime}(t)\right| d t\right\}^{2}$, so that from the relations

$$
\begin{aligned}
& \int_{a}^{b}|| \Delta\left|f^{\prime}(t) \Delta^{-1}-\xi^{\prime}(t)\right| d t=\int_{a}^{b}\left|\eta^{\prime}(t)\right| d t, \\
& \int_{a}^{b}\left|\xi^{\prime}(t)-\mu\right| d t=\int_{a}^{b}\left\{\mu-\xi^{\prime}(t)\right\} d t=\mu \delta-|\Delta|, \\
& \int_{a}^{b}|\mu-| \Delta\left|\delta^{-1}\right| d t=\mu \delta-|\Delta|,
\end{aligned}
$$

follows the relation

$$
\begin{aligned}
\int_{a}^{b}\left|f^{\prime}(t)-\Delta \delta^{-1}\right| d t & =\int_{a}^{b}|| \Delta\left|f^{\prime}(t) \Delta^{-1}-\right| \Delta\left|\delta^{-1}\right| d t \\
& \leqq\left(\mu^{2} \delta^{2}-|\Delta|^{2}\right)^{1 / 2}+2(\mu \delta-|\Delta|) .
\end{aligned}
$$


TheOREM 3.3. If $f_{n}$ is an a.c. function in $B V$ with $\left|f_{n}^{\prime}(t)\right|=\mu_{n}$ for almost all $t$ on $[0,1](n=0,1,2, \cdots)$, then the relation $f_{n}-v \rightarrow f_{0}$ implies $\left\|f_{n}-f_{0}\right\| \rightarrow 0$.

From

$$
\mu_{n}=\int_{0}^{1}\left|f_{n}^{\prime}(t)\right| d t=T_{0}^{1}\left(f_{n}\right) \quad(n=0,1,2, \cdots)
$$

we conclude $\mu_{n} \rightarrow \mu_{0}$. Let $\epsilon$ be any positive number. There exists a partition $\left(0=t_{0}<t_{1}<t_{2}<\cdots<t_{k}=1\right)$ such that $0 \leqq \mu_{0}-\sum_{j=1}^{k}\left|f_{0}\left(t_{j}\right)-f_{0}\left(t_{j-1}\right)\right|<\epsilon$. Letting $\Delta_{n, j}=f_{n}\left(t_{j}\right)-f_{n}\left(t_{j-1}\right)$ and $\delta_{j}=t_{j}-t_{j-1},(n=0,1,2, \cdots ; j=1,2, \cdots, k)$, we have the obvious relation

$$
\sum_{j=1}^{k} \int_{t_{j-1}}^{t_{j}}\left|\Delta_{n, j} \delta_{j}^{-1}-\Delta_{0, j} \delta_{j}^{-1}\right| d t=\sum_{j=1}^{k}\left|\Delta_{n, j}-\Delta_{0, j}\right| \quad(n=0,1,2, \cdots) .
$$

Use of Lemma 3.9 and Lemma 3.3 yields (for $n=0,1,2, \cdots$ )

where

$$
\begin{aligned}
\sum_{j=1}^{k} \int_{t_{j-1}}^{t_{j}}\left|f_{n}^{\prime}(t)-\Delta_{n, j} \delta_{j}^{-1}\right| d t \leqq & \sum_{j=1}^{k}\left(\mu_{n}^{2} \delta_{j}{ }^{2}-\left|\Delta_{n, j}\right|^{2}\right)^{1 / 2} \\
& +\sum_{j=1}^{k} 2\left(\mu_{n} \delta_{j}-\left|\Delta_{n, j}\right|\right) \leqq K_{n},
\end{aligned}
$$

$$
K_{n}=\left\{\mu_{n}^{2}-\left(\sum_{j=1}^{k}\left|\Delta_{n, j}\right|\right)^{2}\right\}^{1 / 2}+2\left\{\mu_{n}-\sum_{j=1}^{k}\left|\Delta_{n, j}\right|\right\} .
$$

Combining these last two relations with the relation obtained by setting $n=0$ in the last relation we conclude

Hence

$$
\begin{aligned}
\int_{0}^{1}\left|f_{n}^{\prime}(t)-f_{0}^{\prime}(t)\right| d t & =\sum_{j=1}^{k} \int_{t_{j-1}}^{t_{j}}\left|f_{n}^{\prime}(t)-f_{0}^{\prime}(t)\right| d t \\
& \leqq K_{n}+K_{0}+\sum_{j=1}^{k}\left|\Delta_{n, j}-\Delta_{0, j}\right| \quad(n=0,1,2, \cdots) .
\end{aligned}
$$

$$
\begin{aligned}
& \limsup _{n \rightarrow \infty} \int_{0}^{1}\left|f_{n}^{\prime}(t)-f_{0}^{\prime}(t)\right| d t \\
& \leqq 2 K_{0}=2\left\{\left(\mu_{0}-\sum_{j=1}^{k}\left|\Delta_{0, j}\right|\right)\left(\mu_{0}+\sum_{j=1}^{k}\left|\Delta_{0, j}\right|\right)\right\}^{1 / 2}+4\left(\mu_{0}-\sum_{j=1}^{k}\left|\Delta_{0, j}\right|\right) \\
& \leqq 2\left(\epsilon \cdot 2 \mu_{0}\right)^{1 / 2}+4 \epsilon,
\end{aligned}
$$

the theorem following from the arbitrariness of $\epsilon$.

4. Transforms of sequences in $B V$. We prove the following lemma. 
LEMMA 4.1. If $\left\{X_{n}\right\}$ is a sequence of functions in $R B V$ satisfying the condition

$$
\left|X_{n}\left(t_{2}\right)-X_{n}\left(t_{1}\right)\right| \leqq M\left|t_{2}-t_{1}\right| \quad\left(0 \leqq t_{1}, t_{2} \leqq 1 ; n=0,1,2, \cdots\right),
$$

and if $\Psi$ is a real function which satisfies a Lipschitz condition on every finite interval, then the relation $\left\|X_{n}-X_{0}\right\| \rightarrow 0$ implies the relation $\left\|\Psi: X_{n}-\Psi: X_{0}\right\| \rightarrow 0$.

Let $[a, b]$ be such that $X_{n}(t)$ is in $[a, b]$ for $0 \leqq t \leqq 1 ; n=0,1,2, \cdots$. Let $M_{1}(>0)$ dominate $\Psi^{\prime}(x)$ for $a \leqq x \leqq b ;$ let $P_{n}=\underset{t}{E}\left[X_{n}^{\prime}(t)>0\right], R_{n}=E\left[X_{n}^{\prime}(t)=0\right]$, $N_{n}=\underset{t}{E}\left[X_{n}^{\prime}(t)<0\right]$ for $n=0,1,2, \cdots$ and denote by $P^{*}, R^{*}, N^{*}$ those points on $[0,1]$ at which the metric density of $P_{0}, R_{0}, N_{0}$ respectively is 1 ; let $\left\{\psi_{p}\right\}$ be a sequence of functions continuous on $[a, b]$ and dominated there by $M_{1}$, such that $\int_{a}^{b} H_{p}(x) d x \rightarrow 0$, where $H_{p}(x)=\left|\Psi^{\prime}(x)-\psi_{p}(x)\right|$ for $x$ on $[a, b]$. Since $\Psi: X_{n}$ satisfies a Lipschitz condition, it becomes clear in the light of Corollary 3.2 that the truth of the theorem is equivalent to showing $F_{n}(t) \rightarrow 0$ for $t$ on $[0,1]$, where $F_{n}$ is defined by

$$
F_{n}(t)=\int_{0}^{t}\left|\Psi^{\prime}\left\{X_{n}(s)\right\} X_{n}^{\prime}(s)-\Psi^{\prime}\left\{X_{0}(s)\right\} X_{0}^{\prime}(s)\right| d s .
$$

To establish this relation we shall prove the fellowing: If $\left\{F_{n}^{*}\right\}$ is any subsequence of $\left\{F_{n}\right\}$, then a subsequence $\left\{G_{n}\right\}$ of $\left\{F_{n}^{*}\right\}$ exists such that $G_{n}(t) \rightarrow 0$ for $t$ on $[0,1]$.

Since the sequence $\left\{F_{n}\right\}$ is comprised of non-decreasing functions which uniformly satisfy a Lipschitz condition, it appears as a corollary of Helly's theorem that there exists a subsequence $\left\{G_{n}\right\}$ of $\left\{F_{n}^{*}\right\}$ and a function $G_{0}$ satisfying a Lipschitz condition such that $G_{n}(t) \rightarrow G_{0}(t)$ for $t$ on $[0,1]$.

Now let $t_{0}$ be any point of $P^{*}$ and denote $\left[t_{0}, s\right]$ by $Q_{s}$ for $0 \leqq s \leqq 1$. From Theorem 3.2 follows the relation

$$
\begin{aligned}
& \left|\int_{t_{0}}^{s}\right| \Psi^{\prime}\left\{X_{n}(t)\right\} X_{n}^{\prime}(t)-\psi_{p}\left\{X_{n}(t)\right\} X_{n}^{\prime}(t)|d t|=\left|\int_{t_{0}}^{s} H_{p}\left\{X_{n}(t)\right\}\right| X_{n}^{\prime}(t)|d t| \\
& =\left|\int_{t_{0}}^{s} H_{p}\left\{X_{n}(t)\right\} X_{n}^{\prime}(t) d t\right|+2 \int_{Q_{s} N_{n}} H_{p}\left\{X_{n}(t)\right\}\left|X_{n}^{\prime}(t)\right| d t \\
& \quad \leqq \int_{a}^{b} H_{p}(x) d x+4 M_{1} M\left|Q_{s} N_{n}\right| \\
& \leqq \int_{a}^{b} H_{p}(x) d x+4 M_{1} M\left\{\left|Q_{s} P_{0} N_{n}\right|+\left|Q_{s}\left(N_{0}+R_{0}\right)\right|\right\} \\
& \quad(n=0,1,2, \cdots ; p=1,2,3, \cdots ; 0 \leqq s \leqq 1) .
\end{aligned}
$$

Thus 


$$
\begin{aligned}
\left|\int_{t_{0}}^{b}\right| \psi_{p}\left\{X_{0}(t)\right\} X_{0}^{\prime}(t)-\Psi^{\prime}\left\{X_{0}(t)\right\} X_{0}^{\prime}(t)|d t| & \\
& \leqq \int_{a}^{b} H_{p}(x) d x+4 M_{1} M\left|Q_{b}\left(N_{0}+R_{0}\right)\right|
\end{aligned}
$$

for $p=1,2,3, \cdots ; 0 \leqq s \leqq 1$; furthermore, since $X_{n}^{\prime}$ converges in measure to $X_{0}^{\prime}$, it may easily be seen that $\left|Q_{8} P_{0} N_{n}\right| \rightarrow 0$ as $n \rightarrow \infty$ and hence that

$$
\begin{aligned}
\limsup \left|\int_{t_{0}}^{0}\right| \Psi^{\prime}\left\{X_{n}(t)\right\} X_{n}^{\prime}(t)-\psi_{p}\left\{X_{n}(t)\right\} X_{n}^{\prime}(t)|d t| \\
\leqq \int_{a}^{b} H_{p}(x) d x+4 M_{1} M\left|Q_{d}\left(N_{0}+R_{0}\right)\right|
\end{aligned}
$$

for $p=1,2,3, \cdots ; 0 \leqq s \leqq 1$. By combining these two relations with the obvious relation

$$
\begin{array}{r}
\limsup _{n \rightarrow \infty}\left|\int_{t_{0}}^{\infty}\right| \psi_{p}\left\{X_{n}(t)\right\} X_{n}^{\prime}(t)-\psi_{p}\left\{X_{0}(t)\right\} X_{0}^{\prime}(t)|d t|=0 \\
(p=1,2,3, \cdots ; 0 \leqq s \leqq 1),
\end{array}
$$

we obtain

$$
\begin{aligned}
\underset{n \rightarrow \infty}{\limsup } & \left|F_{n}(s)-F_{n}\left(t_{0}\right)\right| \\
\quad & =\limsup _{n \rightarrow \infty}\left|\int_{t_{0}}^{e} \Psi^{\prime}\left\{X_{n}(t)\right\} X_{n}^{\prime}(t)-\Psi\left\{X_{0}^{\prime}(t)\right\} X_{0}^{\prime}(t) d t\right| \\
& \leqq 2 \int_{a}^{b} H_{p}(x) d x+8 M_{1} M\left|Q_{s}\left(N_{0}+R_{0}\right)\right| \quad(p=1,2,3, \cdots ; 0 \leqq s \leqq 1)
\end{aligned}
$$

so that upon letting $p \rightarrow \infty$ we conclude

$$
\begin{aligned}
\left|G_{0}(s)-G_{0}\left(t_{0}\right)\right| & =\lim _{n \rightarrow \infty}\left|G_{n}(s)-G_{n}\left(t_{0}\right)\right| \\
& \leqq \limsup _{n \rightarrow \infty}\left|F_{n}(s)-F_{n}\left(t_{0}\right)\right| \leqq 8 M_{1} M\left|Q_{8}\left(N_{0}+R_{0}\right)\right|
\end{aligned}
$$

for $s$ on $[0,1]$. Thus, since $t_{0}$ is a point at which the metric density of $P_{0}$ is 1 ,

$$
\lim _{s \rightarrow t_{0}} \frac{\left|Q_{s}\left(N_{0}+R_{0}\right)\right|}{\left|s-t_{0}\right|}=0
$$

which implies $G_{0}^{\prime}\left(t_{0}\right)=0$.

If $t_{0}$ is a point of $N^{*}$, a similar proof establishes $G_{0}^{\prime}\left(t_{0}\right)=0$; if $t_{0} \varepsilon R^{*}$, then the relation $G_{0}^{\prime}\left(t_{0}\right)=0$ is a consequence of the easily proved inequality

$$
\underset{n \rightarrow \infty}{\lim \sup }\left|F_{n}(s)-F_{n}\left(t_{0}\right)\right| \leqq 2 M_{1} M\left|Q_{*}\left(N_{0}+P_{0}\right)\right| \quad(0 \leqq s \leqq 1) .
$$


Thus $G_{0}^{\prime}(t)=0$ for almost all $t$ on $[0,1]$, and hence $G_{0}(t)=0$ for $t$ on $[0,1]$. This completes the proof.

The following is now readily established.

LEMMA 4.2. If $\left\{X_{n}\right\}$ is a sequence of monotone functions in $R B V$ satisfying the condition

$$
\left|X_{n}\left(t_{2}\right)-X_{n}\left(t_{1}\right)\right| \leqq M\left|t_{2}-t_{1}\right| \quad\left(0 \leqq t_{1}, t_{2} \leqq 1 ; n=0,1,2, \cdots\right),
$$

and if $\Psi$ is a real function which is a.c. on every finite interval, then the relation $\left\|X_{n}-X_{0}\right\| \rightarrow 0$ implies $\left\|\Psi: X_{n}-\Psi: X_{0}\right\| \rightarrow 0$.

To show this, approximate $\Psi^{\prime}$ in the mean with bounded measurable functions and apply Lemma 4.1 and Theorem 3.2. It is of some interest to note that some of the functions which comprise $\left\{X_{n}\right\}$ may be increasing while others are decreasing.

Definition 4.1. Let $u$ be a function on two-space to one-space. If there is a function $A$ on one-space to $n_{1}$-space, a function $B$ on one-space to $n_{2}$ space, and a function $U$ on $\left(n_{1}+n_{2}\right)$-space to one-space such that

(i) $A(x)=\left(A_{1}(x), A_{2}(x), \cdots, A_{n_{1}}(x)\right), B(y)=\left(B_{1}(y), B_{2}(y), \cdots, B_{n_{2}}(y)\right)$ $(-\infty<x, y<\infty)$

(ii) all first partial derivatives of $U\left(\alpha_{1}, \alpha_{2}, \cdots, \alpha_{n_{1}}, \beta_{1}, \beta_{2}, \cdots, \beta_{n_{2}}\right)$ with respect to these arguments are continuous functions on $\left(n_{1}+n_{2}\right)$-space;

(iii) $u(x, y)=U(A(x) \circ B(y))=U\left(A_{1}(x), \cdots, A_{n_{1}}(x), B_{1}(y), \cdots, B_{n_{2}}(y)\right)$ for $-\infty<x, y<\infty$;

(iv) on every finite interval either

$A$ and $B$ are a.c., or

$A$ satisfies a Lipschitz $\dagger$ condition and $B$ is a.c., or

$A$ is a.c. and $B$ satisfies a Lipschitz condition, or

$A$ and $B$ satisfy a Lipschitz condition;

then $u$ is said to be respectively either $\Omega$ or $\Omega_{1}$ or $\Omega_{2}$ or $\Omega_{12}$.

Definition 4.2. Let $\phi$ be a function in $C C$ with $\phi(x+i y)=u(x, y)+i v(x, y)$ for $-\infty<x, y<\infty$; let $f$ be a point in $B V$ with $f=X+i Y$. If the functions $u$ and $v$ are $\Omega$ with $X$ and $Y$ monotone, or $\Omega_{1}$ with $Y$ monotone, or $\Omega_{2}$ with $X$ monotone, or $\Omega_{12}$, then $\phi$ is said to be applicable to $f$.

Definition 4.3. Let $Y$ be a point in $R B V$ and let $u$ be $\Omega$. If $Y$ is monotone or if $u$ is $\Omega_{2}$, then $u$ is said to be applicable $(R)$ to $Y$.

Definition 4.4. If $\phi$ is applicable to $f$ and if

$$
|\phi: f(t \pm)-\phi: f(t)|=T_{\lambda=0}^{1}[\phi\{(1-\lambda) f(t)+\lambda f(t \pm)\}] \quad(0 \leqq t \leqq 1),
$$

then $\phi$ is strictly applicable to $f$.

$\dagger$ Throughout the paper we shall consider definitions which involve purely metric properties of a function on $[a, b]$ to one-space to be generalized in the customary manner to functions on $[a, b]$ to $n$-space. 
Definition 4.5. If $u$ is applicable $(R)$ to $Y$ and if for each $t$ on $[0,1]$ it is true that $u\{t,(1-\lambda) Y(t)+\lambda Y(t \pm)\}$ is monotone in $\lambda$ for $t$ on $[0,1]$, then $u$ is said to be strictly applicable $(R)$ to $Y$.

Definitions 4.2 and 4.4 are formulated to facilitate the discussion of the following problem: Suppose $f_{n}=\left(X_{n}+i Y_{n}\right)-v \rightarrow\left(X_{0}+i Y_{0}\right)=f_{0}$ and suppose $\phi$ in $C C$ with $\phi(x+i y)=u(x, y)+i v(x, y)$ for $-\infty<x, y<\infty$. What conditions on $\phi$ will imply $\phi: f_{n}-v \rightarrow \phi: f_{0}$ ? This is equivalent to asking what condition on $\phi$ will imply

$$
\begin{aligned}
T_{t=0}^{1}\left[u\left\{X_{n}(t), Y_{n}(t)\right\}+i v\left\{X_{n}(t),\right.\right. & \left.\left.Y_{n}(t)\right\}\right] \\
& \rightarrow T_{t=0}^{1}\left[u\left\{X_{0}(t), Y_{0}(t)\right\}+i v\left\{X_{0}(t), Y_{0}(t)\right\}\right] .
\end{aligned}
$$

Definitions 4.3 and 4.5 will be used in connection with convergence in length.

LEMMA 4.3. Let $\sigma$ and $g$ be in $C R$ with $\sigma$ real and non-decreasing and $\sigma(0)=0$, $\sigma(1)=1$. If $f=g: \sigma$ with $f(t \pm)=g\{\sigma(t \pm)\}$ for $t$ on $[0,1]$, then

where

$$
\|g\|=\|f\|+T_{0}^{1}(\Lambda)
$$

$$
\Lambda(t)=2^{\operatorname{sgn} t(t-1)}\left\{T_{\sigma(t-)}^{\sigma(t+)}(g)-|f(t)-f(t-)|-|f(t+)-f(t)|\right\} \quad(0 \leqq t \leqq 1) .
$$

Let $f_{0}$ and $\sigma_{0}$ be defined on $[-2,2]$ as follows:

and let

$$
\begin{array}{llrl}
f_{0}(t) & =f(t), & \sigma_{0}(t)=\sigma(t) & (0 \leqq t \leqq 1), \\
f_{0}(t)=f(1), & \sigma_{0}(t)=\sigma(1) & (1<t \leqq 2), \\
f_{0}(t)=f(0), & \sigma_{0}(t)=\sigma(0) & (-2 \leqq t<0),
\end{array}
$$

$\Lambda_{0}(t)=2^{-1}\left\{T_{\sigma_{0}(t-)}^{\sigma_{0}(t)}(g)-\left|f_{0}(t)-f_{0}(t-)\right|-\left|f_{0}(t+)-f_{0}(t)\right|\right\} \quad(-2 \leqq t \leqq 2)$.

The truth of the lemma is clearly equivalent to showing

$$
T_{0}^{1}(g)=T_{-2}^{2}\left(f_{0}\right)+T_{-2}^{2}\left(\Lambda_{0}\right) .
$$

To do this let $S \equiv\left(0=s_{0}<s_{1}<s_{2}<\cdots<s_{k}=1\right)$ be any partition of $[0,1]$ and let $H\left(s^{\prime}, s^{\prime \prime}\right)$ be defined for $0 \leqq s^{\prime} \leqq s^{\prime \prime} \leqq 1$ by

$$
H\left(s^{\prime}, s^{\prime \prime}\right)=\sum_{j=1}^{q}\left|g\left(p_{j}\right)-g\left(p_{i-1}\right)\right|,
$$

where $p_{0}=s^{\prime}, p_{q}=s^{\prime \prime}$, and $q$ is one more than the number of points of $S$ on $\underset{s}{E}\left[s^{\prime}<s<s^{\prime \prime}\right]$ and $p_{1}<p_{2}<\cdots<p_{q-1}$ are these points (if any). Consider the set

$$
E=\underset{t}{E}\left[H\left(0, \sigma_{0}(t)\right) \leqq T_{-2}^{\ell}\left(f_{0}\right)+T_{-2}^{t}\left(\Lambda_{0}\right)\right]
$$


and denote by $E^{\prime}$ the set of those points which are left-hand limit points $\dagger$ of $E$. Noting that $-1 \varepsilon E^{\prime}$ let $P=\sup E^{\prime}$ and suppose $P<2$ in an attempt to show that $P=2$. Since $P_{\varepsilon} E^{\prime}$ there is a point $P_{1}(>-2)$ of $E$ which is less than $P$ but sufficiently close to $P$ so that $\underset{s}{E}\left[\sigma_{0}\left(P_{1}\right)<s<\sigma_{0}(P-)\right]$ contains no point of $S$. It is likewise clear that there exists a point $P_{2}(>P$ and $<2)$ such that $\underset{s}{E}\left[\sigma_{0}(P+)<s<\sigma_{0}\left(P_{2}\right)\right]$ contains no point of $S$. Now $P_{1} \varepsilon E$ implies

$$
H\left(0, \sigma_{0}\left(P_{1}\right)\right) \leqq T_{-2}^{P_{1}}\left(f_{0}\right)+T_{-2}^{P_{1}}\left(\Lambda_{0}\right)
$$

and from the fact that $\Lambda_{0}$ vanishes everywhere on $[-2,2]$ except for a denumerable set it follows that

$T_{\sigma(P-)}^{\sigma(P+)}(g)-\left|f_{0}(P)-f_{0}(P-)\right|-\left|f_{0}(P+)-f_{0}(P)\right| \leqq T_{P_{1}}^{R}\left(\Lambda_{0}\right) \quad\left(P<R<P_{2}\right)$

which implies

$$
\begin{aligned}
& H\left(\sigma_{0}(P-), \sigma_{0}(P+)\right) \leqq T_{\sigma_{0}(P-)}^{\sigma_{0}(P+)}(g) \\
& \quad \leqq\left|f_{0}(P)-f_{0}(P-)\right|+\left|f_{0}(P+)-f_{0}(P)\right|+T_{P_{1}}^{R}\left(\Lambda_{0}\right) \quad\left(P<R<P_{2}\right) .
\end{aligned}
$$

Furthermore the fact that $E\left[\sigma_{0}\left(P_{1}\right)<s<\sigma_{0}(P-)\right]+\underset{s}{E}\left[\sigma_{0}(P+)<s<\sigma_{0}\left(P_{2}\right)\right]$ contains no point of $S$ combines with the hypothesis of the lemma to yield

$$
\begin{aligned}
& H\left(\sigma_{0}\left(P_{1}\right), \sigma_{0}(P-)\right)+H\left(\sigma_{0}(P+), \sigma_{0}(R)\right) \\
& =\left|f_{0}(P-)-f_{0}\left(P_{1}\right)\right|+\left|f_{0}(R)-f_{0}(P+)\right| \cdot\left(P<R<P_{2}\right),
\end{aligned}
$$

so that upon adding (1), (2), and (3) we obtain, for $P<R<P_{2}$,

$$
\begin{aligned}
H\left(0, \sigma_{0}(R)\right) \leqq & H\left(0, \sigma_{0}\left(P_{1}\right)\right)+H\left(\sigma_{0}\left(P_{1}\right), \sigma_{0}(P-)\right) \\
& +H\left(\sigma_{0}(P-), \sigma_{0}(P+)\right)+H\left(\sigma_{0}(P+), \sigma_{0}(R)\right) \\
\leqq & T_{-2}^{P_{1}}\left(f_{0}\right)+\left|f_{0}(P-)-f_{0}\left(P_{1}\right)\right|+\left|f_{0}(P)-f_{0}(P-)\right| \\
& +\left|f_{0}(P+)-f_{0}(P)\right|+\left|f_{0}(R)-f_{0}(P+)\right|+T_{-2}^{P_{1}}\left(\Lambda_{0}\right)+T_{P_{1}}^{R}\left(\Lambda_{0}\right) \\
\leqq & T_{-2}^{R}\left(f_{0}\right)+T_{-2}^{R}\left(\Lambda_{0}\right) .
\end{aligned}
$$

This establishes $P_{2}$ as a point of $E^{\prime}$ which is a contradiction proving $2=\sup E^{\prime}$ and hence that

$$
\sum_{j=1}^{k}\left|g\left(s_{j}\right)-g\left(s_{j-1}\right)\right|=H(0,1) \leqq T_{-2}^{2}\left(f_{0}\right)+T_{-2}^{2}\left(\Lambda_{0}\right),
$$

which implies

since $S$ was arbitrary.

$$
T_{0}^{1}(g) \leqq T_{-2}^{2}\left(f_{0}\right)+T_{-2}^{2}\left(\Lambda_{0}\right)
$$

$\dagger$ We define $x_{0}$ as a left-hand limit point of $E$ if every interval $\left[x, x_{0}\right]$, where $x<x_{0}$, contains a point of $E$ as an inner point. 
Now let $t_{1}, t_{2}, t_{3}, \cdots$ be a denumerable set of points on $[0,1]$ which include all points of discontinuity of $\Lambda_{0}$ and define $\Lambda_{n}(t)=\Lambda_{0}(t)$ for $t=t_{1}, t_{2}, \cdots, t_{n}$ and $\Lambda_{n}(t)=0$ for all other $t$ on [-2,2]. Clearly $\Lambda_{n}(t) \rightarrow \Lambda_{0}(t)$ for all $t$ on [-2,2] and inasmuch as

$$
T_{\sigma_{0}\left(t^{\prime}\right)}^{\sigma_{0}\left(t^{\prime \prime}\right)}(g) \geqq T_{t^{\prime}}^{t^{\prime \prime}\left(f_{0}\right) \quad\left(-2 \leqq t^{\prime} \leqq t^{\prime \prime} \leqq 2\right),}
$$

it follows by induction that

$$
T_{\sigma_{0}\left(t^{\prime}\right)}^{\sigma_{0}\left(t^{\prime \prime}\right)}(g) \geqq T_{t^{\prime}}^{t^{\prime \prime}}\left(f_{0}\right)+T_{t^{\prime}}^{t^{\prime \prime}}\left(\Lambda_{n}\right)
$$

for $t^{\prime} \leqq t^{\prime \prime}$ with $t^{\prime}$ and $t^{\prime \prime}$ both in the set obtained by deleting the points $t_{1}, t_{2}, t_{3}, \cdots$ from $[-2,2]$. This of course implies

$$
T_{0}^{1}(g) \geqq T_{-2}^{2}\left(f_{0}\right)+T_{-2}^{2}\left(\Lambda_{n}\right)
$$

whence, by semi-continuity

$$
T_{0}^{1}(g) \geqq T_{-2}^{2}\left(f_{0}\right)+T_{-2}^{2}\left(\Lambda_{0}\right)
$$

and the proof of the lemma is complete.

LEMMA 4.4. Let $f=X+i Y$ be in $B V$ and denote $T_{0}{ }^{1}(f)$ by $\mu$. Let $\sigma$ be a nondecreasing function in $R B V$ such that $\sigma(0)=0, \sigma(1)=1$ and $\mu \sigma(t)=T_{0}{ }^{t}(f)$ for $t$ on $[0,1]$. There exists a function $g$ in $B V$ having the following properties:

(i) $f=g: \sigma$ with $\|f\|=\|g\|$;

(ii) $\left|g\left(s_{2}\right)-g\left(s_{1}\right)\right| \leqq \mu\left|s_{2}-s_{1}\right|,\left(0 \leqq s_{1}, s_{2} \leqq 1\right)$, the sign of equality holding if $\sigma(t-) \leqq s_{1}, s_{2} \leqq \sigma(t)$ or $\sigma(t) \leqq s_{1}, s_{2} \leqq \sigma(t+)$, where $0 \leqq t \leqq 1$;

(iii) if $\phi$ is any function in CC which is applicable to $g$, then

where

$$
\|\phi: g\|=\|\phi: f\|+T_{0}^{1}(\Lambda) \text {, }
$$

$$
\begin{aligned}
\Lambda(t) & =2^{\operatorname{sgn} t(t-1)}\left\{T_{\lambda=0}^{1}[\phi\{\lambda f(t-)+(1-\lambda) f(t)\}]-|\phi: f(t)-\phi: f(t-1)|\right. \\
& \left.+T_{\lambda=0}^{1}[\phi\{\lambda f(t)+(1-\lambda) f(t+)\}]-|\phi: f(t+)-\phi: f(t)|\right\}(0 \leqq t \leqq 1) .
\end{aligned}
$$

Define $g=\xi+i \eta$ as follows. Let $s_{0}$ be any point on $[0,1]$ and let $t_{0}=\inf \underset{t}{E}\left[\sigma(t) \geqq s_{0}\right]$. Now $\sigma\left(t_{0}-\right) \leqq s_{0} \leqq \sigma\left(t_{0}+\right)$ and we define

$$
\begin{aligned}
& g\left(s_{0}\right)=f\left(t_{0}\right) \text { if } s_{0}=\sigma\left(t_{0}\right) ; \\
& g\left(s_{0}\right)=\frac{f\left(t_{0}-\right)\left\{\sigma\left(t_{0}\right)-s_{0}\right\}+f\left(t_{0}\right)\left\{s_{0}-\sigma\left(t_{0}-\right)\right\}}{\sigma\left(t_{0}\right)-\sigma\left(t_{0}-\right)} \text { if } \sigma\left(t_{0}-\right) \leqq s_{0}<\sigma\left(t_{0}\right) ; \\
& g\left(s_{0}\right)=\frac{f\left(t_{0}\right)\left\{\sigma\left(t_{0}+\right)-s\right\}+f\left(t_{0}+\right)\left\{s_{0}-\sigma\left(t_{0}\right)\right\}}{\sigma\left(t_{0}+\right)-\sigma\left(t_{0}\right)} \text { if } \sigma\left(t_{0}\right)<s_{0} \leqq \sigma\left(t_{0}+\right) .
\end{aligned}
$$


As a consequence of

$$
\left|f\left(t^{\prime \prime}\right)-f\left(t^{\prime}\right)\right| \leqq \mu\left|\sigma\left(t^{\prime \prime}\right)-\sigma\left(t^{\prime}\right)\right| \quad\left(0 \leqq t^{\prime}, t^{\prime \prime} \leqq 1\right),
$$

it is easily verified that $g\{\sigma(t)\}=f(t)$ and $g\{\sigma(t \pm)\}=f(t \pm)$ for $t$ on $[0,1]$. Combining these last two relations with the definition of $g$ and the relation

$$
|f(t \pm)-f(t)|=\mu|\sigma(t \pm)-\sigma(t)| \quad(0 \leqq t \leqq 1),
$$

we obtain

$$
\left|g\left(s_{2}\right)-g\left(s_{1}\right)\right|=\mu^{\mu}\left|s_{2}-s_{1}\right|
$$

if $\sigma(t-) \leqq s_{1}, s_{2} \leqq \sigma(t)$ or $\sigma(t) \leqq s_{1}, s_{2} \leqq \sigma(t+)$, where $0 \leqq t \leqq 1$. Hence

$$
\begin{aligned}
T_{\sigma(t)}^{\sigma(t+)}(g) & =\mu|\sigma(t)-\sigma(t-)|+\mu|\sigma(t+)-\sigma(t)| \\
& =|f(t)-f(t-)|+|f(t+)-f(t)|
\end{aligned}
$$

while, on the other hand, from Lemma 3.8 it follows that

$$
\begin{aligned}
\Lambda(t)= & 2^{\operatorname{sgn} t(t-1)}\left[T_{\sigma(t-)}^{\sigma(t)}(\phi: g)-\mid \phi: f(t)\right. \\
& -\phi: f(t-) \mid \\
& \left.\quad+T_{\sigma(t)}^{\sigma(t+)}(\phi: g)-|\phi: f(t+)-\phi: f(t)|\right] \\
= & 2^{\operatorname{sgn} t(t-1)}\left[T_{\sigma(t-)}^{\sigma(t+)}(\phi: g)-|\phi: f(t)-\phi: f(t-)|-|\phi: f(t+)-\phi: f(t)|\right] .
\end{aligned}
$$

Viewing the last two relations in the light of Lemma 4.3 establishes $\|g\|=\|f\|$ and $\|\phi: g\|=\|\phi: f\|+T_{0}{ }^{1}(\Lambda)$. It also follows that monotonicity of $X$ or $Y$ implies monotonicity of $\xi$ or $\eta$ respectively so that from Definition 4.2 we conclude $\phi$ is applicable to $g$.

To complete the proof of (ii) let $s_{1}^{\prime} \leqq s_{2}^{\prime}$ be any two numbers on $[0,1]$ and let $t_{1}^{\prime}=\inf \underset{t}{E}\left[\sigma(t) \geqq s_{1}^{\prime}\right]$ and $t_{2}^{\prime}=\sup \underset{t}{E}\left[\sigma(t) \leqq s_{2}^{\prime}\right]$. Now $t_{1}^{\prime} \leqq t_{2}^{\prime}$. If $t_{1}^{\prime}=t_{2}^{\prime}$ then $\sigma\left(t_{1}^{\prime}-\right) \leqq s_{1}^{\prime} \leqq s_{2}^{\prime} \leqq \sigma\left(t_{1}^{\prime}+\right)$, so that the relation (2) implies the inequality $\left|g\left(s_{2}^{\prime}\right)-g\left(s_{1}^{\prime}\right)\right| \leqq \mu\left|s_{2}^{\prime}-s_{1}^{\prime}\right|$. However, if $t_{1}^{\prime}<t_{2}^{\prime}$ then

$$
\sigma\left(t_{1}^{\prime}-\right) \leqq s_{1}^{\prime} \leqq \sigma\left(t_{1}^{\prime}+\right) \leqq \sigma\left(t_{2}^{\prime}-\right) \leqq s_{2}^{\prime} \leqq \sigma\left(t_{2}^{\prime}+\right),
$$

so that (1) and (2) yield

$$
\begin{aligned}
\left|g\left(s_{2}^{\prime}\right)-g\left(s_{1}^{\prime}\right)\right| \leqq & \left|g\left\{\sigma\left(t_{1}^{\prime}+\right)\right\}-g\left(s_{1}^{\prime}\right)\right|+\left|g\left\{\sigma\left(t_{2}^{\prime}-\right)\right\}-g\left\{\sigma\left(t_{1}^{\prime}+\right)\right\}\right| \\
& +\left|g\left(s_{2}^{\prime}\right)-g\left\{\sigma\left(t_{2}^{\prime}-\right)\right\}\right| \\
\leqq & \mu\left\{\sigma\left(t_{1}^{\prime}+\right)-s_{1}^{\prime}\right\}+\left|f\left(t_{2}^{\prime}-\right)-f\left(t_{1}^{\prime}+\right)\right|+\mu\left\{s_{2}^{\prime}-\sigma\left(t_{2}^{\prime}-\right)\right\} \\
\leqq & \mu\left\{\sigma\left(t_{1}^{\prime}+\right)-s_{1}^{\prime}\right\}+\mu\left\{\sigma\left(t_{2}^{\prime}-\right)-\sigma\left(t_{1}^{\prime}+\right)\right\} \\
& +\mu\left\{s_{2}^{\prime}-\sigma\left(t_{2}^{\prime}-\right)\right\}=\mu\left(s_{2}^{\prime}-s_{1}^{\prime}\right) .
\end{aligned}
$$

The truth of (ii) is now apparent and the proof of the lemma is complete. 
We introduce here the notion of pseudo-absolute continuity.

Definition 4.6. A function $f$ defined on $[a, b]$ is said to be pseudoabsolutely continuous there if corresponding to every $\epsilon(>0)$ there exists a $\delta(>0)$ and a finite point set $E$ such that if $\left\{\left[a_{n}, b_{n}\right]\right\}$ is any denumerable set of non-overlapping intervals on $[a, b]$ with $E \cdot \sum_{n=1}^{\infty}\left[a_{n}, b_{n}\right]$ empty and $\sum_{n=1}^{\infty}\left|b_{n}-a_{n}\right|<\delta$, then $\sum_{n=1}^{\infty}\left|f\left(b_{n}\right)-f\left(a_{n}\right)\right|<\epsilon$.

We observe that a pseudo-a.c. function is of b.v. and is expressible as the sum of an a.c. function and a singular function of the saltus type (see Definition 6.2 below).

LEMMA 4.5. If $f$ is a pseudo-absolutely continuous point in $B V$ and if $\phi$ is any function in CC which is applicable to $f$, then $\phi: g$ is a pseudo-absolutely continuous point in $B V$.

There is no loss in generality in assuming $\phi$ to be real valued.

Let $X+i Y=f$. From Definitions 4.1 and 4.2 there exist functions $A$ and $B$ on one-space to $n_{1}$ - and $n_{2}$-spaces respectively which are a.c. on every finite interval and a function $U$ on $n_{3}$-space $\left(n_{3}=n_{1}+n_{2}\right)$ to one-space, all of whose first partial derivatives are continuous, such that

$$
\phi(x+i y)=U\{A(x) \circ B(y)\} \quad(-\infty<x, y<\infty)
$$

with $A$ satisfying a Lipschitz condition on every finite interval if $X$ is not monotone and $B$ satisfying a Lipschitz condition on every finite interval if $Y$ is not monotone. Let

$$
C(t)=A\{X(t)\} \circ B\{Y(t)\} \quad(0 \leqq t \leqq 1) .
$$

Readily seen is the pseudo-absolute continuity $\dagger$ of $C$. Let $S$ be a sphere in $n_{3}$-space which includes the range of $C$. Now $U$ satisfies a Lipschitz condition on $S$; i.e., there exists a constant $M(>0)$ such that if $\gamma$ and $\gamma^{\prime}$ are any two points of $S$, then

$$
\left.\left|U\left(\gamma^{\prime}\right)-U(\gamma)\right| \leqq M \text { Euclidean distance between } \gamma \text { and } \gamma^{\prime}\right\},
$$

so that, since $\phi: f(t)=U\{C(t)\}$ for $t$ on [0,1], the pseudo-absolute continuity of $\phi: f$ becomes apparent.

Since a continuous pseudo-absolutely continuous function is a.c. we have

COROLLARY 4.1. If $f$ is an a.c. point in $B V$ and if $\phi$ is any function in $C C$ which is applicable to $f$, then $\phi: g$ is an a.c. point in $B V$.

LEMMA 4.6. If $\left\{g_{n}\right\}$ is a sequence of points in $B V$ satisfying the relation

$$
\left|g_{n}\left(t_{2}\right)-g_{n}\left(t_{1}\right)\right| \leqq M\left|t_{2}-t_{1}\right| \quad\left(0 \leqq t_{1}, t_{2} \leqq 1 ; n=0,1,2, \cdots\right) .
$$

† See footnote on page 59. 
and if $\phi$ is any function in CC which is applicable to $g_{n}$ for $n=0,1,2, \ldots$, then the relation $\left\|g_{n}-g_{0}\right\| \rightarrow 0$ implies $\left\|\phi: g_{n}-\phi: g_{0}\right\| \rightarrow 0$.

There is no loss in generality in assuming $\phi$ real valued. Let $\left\{g_{n, 1}\right\}$ be any subsequence of $\left\{g_{n}\right\}$ wherein $g_{0,1}=g_{0}$. To prove $\left\|\phi: g_{n}-\phi: g_{0}\right\| \rightarrow 0$ it is merely necessary to establish the existence of a sequence $\left\{g_{n, 2}\right\}$ which is a subsequence of $\left\{g_{n, 1}\right\}$, such that $\left\|\phi: g_{n, 2}-\phi: g_{0}\right\| \rightarrow 0$. First we note that of the sequence $\left\{g_{n, 1}\right\}$ there exists a subsequence $\left\{g_{n, 2}\right\}$ wherein $g_{0,2}=g_{0}$ and $X_{n}+i Y_{n}=g_{n, 2}$ for $n=0,1,2, \cdots$, which enjoys one of the following four properties:

(i) $X_{1}$ and $Y_{1}$ are both not monotone;

(ii) $X_{n}$ is monotone $(n=0,1,2, \cdots)$ and $Y_{1}$ is not;

(iii) $X_{n}$ and $Y_{n}$ are monetone $(n=0,1,2, \cdots)$;

(iv) $X_{1}$ is not monotone and $Y_{n}$ is monotone $(n=0,1,2, \cdots)$.

Now since $\phi$ is applicable to $\left\{g_{1,2}\right\}$, there exist functions $A$ and $B$ on onespace to $n_{1}$ - and $n_{2}$-spaces respectively and a real function $U$ defined on $n_{3}$-space $\left(n_{3}=n_{1}+n_{2}\right)$, all of whose first partial derivatives are continuous, such that

$$
\phi(x+i y)=U\{A(x) \circ B(y)\} \quad(-\infty<x, y<\infty)
$$

and such that $C_{n}$ defined by $C_{n}(t)=A\left\{X_{n}(t)\right\} \circ B\left\{Y_{n}(t)\right\}(0 \leqq t \leqq 1)$, is an a.c. function on $[0,1]$ to $n_{3}$-space not only for $n=1$, but for $n=0,1,2, \cdots$. Thus upon defining

$$
\begin{aligned}
\left(C_{n, 1}(t), C_{n, 2}(t), \cdots, C_{n, n_{3}}(t)\right)=C_{n}(t) \text { and } H_{n}(t)=U\left\{C_{n}(t)\right\} \\
\\
(0 \leqq t \leqq 1, n=0,1,2, \cdots),
\end{aligned}
$$

we conclude $H_{n}$ is an a.c. point in $R B V$ with $H_{n}=\phi: g_{n, 2}$ for $n=0,1,2, \cdots$. Also follows the existence of continuous functions $D_{1}, D_{2}, \cdots, D_{n_{3}}$ on $n_{3}$ space to one-space such that (for $n=0,1,2, \cdots$ )

$$
H_{n}^{\prime}(t)=\sum_{p=1}^{n_{3}} D_{p}\left\{C_{n}(t)\right\} C_{n, p}^{\prime}(t) \quad \text { (almost all } t \text { on }[0,1] \text { ). }
$$

Since $g_{0}$ is continuous, we conclude $\left(X_{n}+i Y_{n}\right)-u v \rightarrow\left(X_{0}+i Y_{0}\right)$ and hence $C_{n}(t) \rightarrow C_{0}(t)$ uniformly for $t$ on $[0,1]$, so that

$$
\lim _{n \rightarrow \infty} D_{p}\left\{C_{n}(t)\right\}=D_{p}\left\{C_{0}(t)\right\} \quad\left(p=1,2, \cdots, n_{3}\right)
$$

uniformly for $t$ on $[0,1]$. Combining this with the relation

$$
\lim _{n \rightarrow \infty} \int_{0}^{1}\left|C_{n, p}^{\prime}(t)-C_{0, p}^{\prime}(t)\right| d t=0 \quad\left(p=1,2, \cdots, n_{3}\right),
$$


which is a corollary of Lemma 4.1 and Lemma 4.2, yields

$$
T_{0}^{1}\left(H_{n}-H_{0}\right)=\int_{0}^{1}\left|H_{n}^{\prime}(t)-H_{0}^{\prime}(t)\right| d t \rightarrow 0 .
$$

Hence, since $H_{n}(0) \rightarrow H_{0}(0)$, we conclude $\left\|\phi: g_{n, 2}-\phi: g_{0}\right\| \rightarrow 0$. This completes the proof.

With this background we now turn to the proofs of the following two theorems.

THEOREM 4.1. If $\phi$ is applicable to $f_{n}$ for $n=0,1,2, \cdots$ and in addition if $\phi$ is strictly applicable to $f_{0}$, then the relation $f_{n}-v \rightarrow f_{0}$ implies

$$
\phi: f_{n}-v \rightarrow \phi: f_{0} .
$$

TheOREM 4.2. If $\phi$ is applicable to $f_{n}$ for $n=0,1,2, \cdots$, then the relation $f_{n}-u v \rightarrow f_{0}$ implies

$$
\phi: f_{n}-u v \rightarrow \phi: f_{0} .
$$

Let $\mu_{n}=T_{0}{ }^{1}\left(f_{n}\right),(n=0,1,2, \cdots)$. From Lemma 4.4 we conclude the existence (for $n=0,1,2, \cdots$ ) of functions $g_{n}$ and $\sigma_{n}$ in $C R$ having the following properties:

(i) $\sigma_{n}$ is a non-decreasing function with $\sigma_{n}(0)=0, \sigma_{n}(1)=1$ and $\mu_{n} \sigma_{n}(t)$ $=T_{0}{ }^{t}\left(f_{n}\right)$ for $0 \leqq t \leqq 1$;

(ii) $f_{n}=g_{n}: \sigma_{n}$ with $\left\|f_{n}\right\|=\left\|g_{n}\right\|$;

(iii) $\left|g_{n}\left(s_{2}\right)-g_{n}\left(s_{1}\right)\right| \leqq \mu_{n}\left|s_{2}-s_{1}\right|,\left(0 \leqq s_{1}, s_{2} \leqq 1\right)$, the sign of equality holding if $\sigma_{n}(t-) \leqq s_{1}, s_{2} \leqq \sigma_{n}(t)$ or $\sigma_{n}(t) \leqq s_{1}, s_{2} \leqq \sigma_{n}(t+)$, where $0 \leqq t \leqq 1$;

(iv) $\phi$ is applicable to $g_{n}$ and

$$
\left\|\phi: g_{n}\right\|=\left\|\phi: f_{n}\right\|+T_{0}^{1}\left(\Lambda_{n}\right)
$$

where

$$
\begin{aligned}
\Lambda_{n}(t)= & 2^{\operatorname{sgn} t(t-1)}\left\{T_{\lambda=0}^{1}\left[\phi\left\{\lambda f_{n}(t-)+(1-\lambda) f_{n}(t)\right\}\right]-\left|\phi: f_{n}(t)-\phi: f_{n}(t-)\right|\right. \\
& \left.+T_{\lambda=0}^{1}\left[\phi\left\{\lambda f_{n}(t)+(1-\lambda) f_{n}(t+)\right\}\right]-\left|\phi: f_{n}(t+)-\phi: f_{n}(t)\right|\right\} \\
& (0 \leqq t \leqq 1) .
\end{aligned}
$$

We divide the remainder of the proof of Theorems 4.1 and 4.2 into three parts.

PART I. $\phi: g_{n}$ is a.c. for each $n=0,1,2, \cdots$ and $\left\|\phi: g_{n}-\phi: g_{0}\right\| \rightarrow 0$.

From (i) and Lemma 3.1 follows the relation, $\mu_{n} \sigma_{n}(t) \rightarrow \mu_{0} \sigma_{0}(t)$ for $t$ on $[0,1]$ so that

$$
\left|g_{n}\left(\sigma_{n}(t)\right)-g_{n}\left(\sigma_{0}(t)\right)\right| \leqq\left|\mu_{n} \sigma_{n}(t)-\mu_{n} \sigma_{0}(t)\right| \rightarrow 0
$$


since $\mu_{n} \rightarrow \mu_{0}$. From (ii) follows the relation $g_{n}\left\{\sigma_{n}(t)\right\} \rightarrow g_{0}\left\{\sigma_{0}(t)\right\}$ for $t$ on $[0,1]$, so that upon combining the above relations we conclude

$$
g_{n}\left\{\sigma_{0}(t)\right\} \rightarrow g_{0}\left\{\sigma_{0}(t)\right\} \quad(0 \leqq t \leqq 1) .
$$

Now let $s_{0}$ be any point on $[0,1]$ and note that there exists a point $t_{0}$ on $[0,1]$ for which $\sigma_{0}\left(t_{0}-\right) \leqq s_{0} \leqq \sigma_{0}\left(t_{0}+\right)$, so that either $s_{0}=\sigma_{0}\left(t_{0}\right)$, or $\sigma_{0}\left(t_{0}-\right) \leqq s_{0}<\sigma_{0}\left(t_{0}\right)$, or $\sigma_{0}\left(t_{0}\right)<s_{0} \leqq \sigma_{0}\left(t_{0}+\right)$. If $s_{0}=\sigma_{0}\left(t_{0}\right)$ then follows immediately the conclusion $g_{n}\left(s_{0}\right) \rightarrow g_{0}\left(s_{0}\right)$. Supposing now that $\sigma_{0}\left(t_{0}-\right) \leqq s_{0}<\sigma_{0}\left(t_{0}\right)$, let $0<t_{1}<t_{2}<t_{3}<\cdots$ with $t_{p} \rightarrow t_{0}$ and denote by $P$ any limit point of the sequence $\left\{g_{n}\left(s_{0}\right)\right\}$. Also let $s_{0}^{\prime}=\sigma_{0}\left(t_{0}-\right)$ and $s_{0}^{\prime \prime}=\sigma_{0}\left(t_{0}\right), P_{0}^{\prime}=g_{0}\left(s_{0}^{\prime}\right), P_{0}=g_{0}\left(s_{0}\right)$, and $P_{0}^{\prime \prime}=g_{0}\left(s_{0}^{\prime \prime}\right)$. Thus

$$
\begin{aligned}
\left|g_{n}\left(s_{0}\right)-g_{n}\left\{\sigma_{0}\left(t_{p}\right)\right\}\right| & \leqq \mu_{n}\left\{s_{0}-\sigma_{0}\left(t_{p}\right)\right\}, \\
\left|g_{n}\left(s_{0}^{\prime \prime}\right)-g_{n}\left(s_{0}\right)\right| & \leqq \mu_{n}\left\{s_{0}^{\prime \prime}-s_{0}\right\} \quad(n=0,1,2, \cdots ; p=1,2,3, \cdots) .
\end{aligned}
$$

Letting $n \rightarrow \infty$ and then $p \rightarrow \infty$ and then using (iii) establishes

$$
\begin{aligned}
& \left|P-P_{0}^{\prime}\right| \leqq \mu_{0}\left\{s_{0}-s_{0}^{\prime}\right\}=\left|P_{0}-P_{0}^{\prime}\right| \\
& \left|P_{0}^{\prime \prime}-P\right| \leqq \mu_{0}\left\{s_{0}^{\prime \prime}-s_{0}\right\}=\left|P_{0}^{\prime \prime}-P_{0}\right| .
\end{aligned}
$$

Adding and using (iii) again yields

$$
\left|P_{0}^{\prime \prime}-P_{0}^{\prime}\right| \leqq\left|P-P_{0}^{\prime}\right|+\left|P_{0}^{\prime \prime}-P\right| \leqq \mu_{0}\left(s_{0}^{\prime \prime}-s_{0}^{\prime}\right)=\left|P_{0}^{\prime \prime}-P_{0}^{\prime}\right|
$$

which implies equality in the last three relations which in turn implies $P_{0}=P$. Thus $g_{n}\left(s_{0}\right) \rightarrow g_{0}\left(s_{0}\right)$ if $\sigma_{0}\left(t_{0}-\right) \leqq s_{0}<\sigma_{0}\left(t_{0}\right)$. A similar proof of this relation holds if $\sigma_{0}\left(t_{0}\right)<s_{0} \leqq \sigma_{0}\left(t_{0}+\right)$, so that finally it is established that $g_{n}(s) \rightarrow g_{0}(s)$ for $s$ on $[0,1]$.

From (iii) we conclude $g_{n}$ is a.c. with $\left|g_{n}^{\prime}(s)\right| \leqq \mu_{n}$, for $0 \leqq s \leqq 1$; $n=0,1,2, \cdots$. Hence (for $n=0,1,2, \cdots$ )

$$
\int_{0}^{1}\left|\mu_{n}-\right| g_{n}^{\prime}(s)|| d s=\int_{0}^{1}\left\{\mu_{n}-\left|g_{n}^{\prime}(s)\right|\right\} d s=0,
$$

so that $\mu_{n}=\left|g_{n}^{\prime}(s)\right|$ for almost all $s$ on $[0,1]$. Applying Theorem 3.3 yields $\left\|g_{n}-g_{0}\right\| \rightarrow 0$. Letting $M(>0)$ be such that $\mu_{n}<M$ for $n=0,1,2, \cdots$ we see from (iii) that

$$
\left|g_{n}\left(s_{2}\right)-g_{n}\left(s_{1}\right)\right| \leqq M\left|s_{2}-s_{1}\right| \quad\left(0 \leqq s_{1}, s_{2} \leqq 1 ; n=0,1,2, \cdots\right),
$$

so that Lemma 4.6 and Corollary 4.1 complete the proof of Part I.

ParT II. (Proof of Theorem 4.1.) Since $\phi$ is strictly applicable to $f_{0}$ it is apparent from (iv) that $\Lambda_{0}(t)=0,(0 \leqq t \leqq 1)$, and hence

$$
\left\|\phi: g_{n}\right\| \geqq\left\|\phi: f_{n}\right\| \quad(n=0,1,2, \cdots),
$$


the equality holding if $n=0$. Thus $\phi: f_{n}$ is in $B V$ for $n=0,1,2, \ldots$ and further, since $\phi: f_{n}(t) \rightarrow \phi: f_{0}(t)$ for $t$ on $[0,1]$, we have

$$
\begin{aligned}
0 & =\limsup _{n \rightarrow \infty}\left\|\phi: g_{n}-\phi: g_{0}\right\| \geqq \underset{n \rightarrow \infty}{\lim \sup }\left\|\phi: g_{n}\right\|-\left\|\phi: g_{0}\right\| \\
& \geqq \limsup _{n \rightarrow \infty}\left\|\phi: f_{n}\right\|-\left\|\phi: f_{0}\right\| \geqq \liminf _{n \rightarrow \infty}\left\|\phi: f_{n}\right\|-\left\|\phi: f_{0}\right\| \geqq 0
\end{aligned}
$$

by the semi-continuity property of total variation.

The proof of Part II is now complete.

PART III. (Proof of Theorem 4.2.) Since $\left\|\phi: g_{n}\right\| \geqq\left\|\phi: f_{n}\right\|$ we conclude as before that $\phi: f_{n}$ is a point of $B V$ for each $n=0,1,2, \ldots$. Since, however, $f_{n}-u v \rightarrow f_{0}$ it becomes clear that in this case

$$
f_{n}(t \pm) \rightarrow f_{0}(t \pm) \text { uniformly for } t \text { on }[0,1] \text {, }
$$

which used in connection with Lemma 4.6 readily establishes

$$
\Lambda_{n}(t) \rightarrow \Lambda_{0}(t) \quad(0 \leqq t \leqq 1),
$$

so that $\lim \inf _{n \rightarrow \infty} T_{0}{ }^{1}\left(\Lambda_{n}\right)-T_{0}{ }^{1}\left(\Lambda_{0}\right) \geqq 0$. Hence

$$
\begin{aligned}
0 & =\underset{n \rightarrow \infty}{\limsup }\left\|\phi: g_{n}-\phi: g_{0}\right\| \geqq \underset{n \rightarrow \infty}{\limsup }\left\|\phi: g_{n}\right\|-\left\|\phi: g_{0}\right\| \\
& \geqq \limsup _{n \rightarrow \infty}\left\|\phi: f_{n}\right\|-\left\|\phi: f_{0}\right\|+\underset{n \rightarrow \infty}{\liminf } T_{0}^{1}\left(\Lambda_{n}\right)-T_{0}^{1}\left(\Lambda_{0}\right) \\
& \geqq \limsup _{n \rightarrow \infty}\left\|\phi: f_{n}\right\|-\left\|\phi: f_{0}\right\| \geqq \liminf _{n \rightarrow \infty}\left\|\phi: f_{n}\right\|-\left\|\phi: f_{0}\right\| \geqq 0 .
\end{aligned}
$$

Since $\phi: f_{n}(t) \rightarrow \phi: f_{0}(t)$ uniformly for $t$ on $[0,1]$, the proof of Part III is now complete.

As a corollary we have

Corollary 4.2. If the function $\phi$ is in CC with $\phi(x+i y)=u(x, y)+i v(x, y)$ for $-\infty<x, y<\infty$, where $u$, $v$ have continuous first partial derivatives, then the relation $f_{n}-v \rightarrow f_{0}$ with $f_{0}$ continuous implies

$$
\phi: f_{n}-v \rightarrow \phi: f_{0} .
$$

If the transformation is that of raising to a positive integer power, Theorems 4.1 and 4.2 lead to

COROLLARY 4.3. Let $k$ be a positive integer. If $f_{n}-v \rightarrow f_{0}$ and if corresponding to each $t$ on $[0,1]$ there is a ray through the origin (of the complex plane) on which lie the points $f_{0}(t), f_{0}(t+)$, and $f_{0}(t-)$, then

$$
f_{n}^{k}-v \rightarrow f_{0}^{k} .
$$

CoRolla.RY 4.4. If $f_{n}-u v \rightarrow f_{0}$, then $f_{n}^{k}-u v \rightarrow f_{0}^{k}$. 
Before concluding this section the following remark seems in order. If $\phi$ is in $C C$ and satisfies a Lipschitz condition on every bounded set in the complex plane, then $f_{\varepsilon} B V$ implies $\phi: f_{\varepsilon} B V$. Hence it is natural to inquire into the truth of the following statement.

If $\phi_{\varepsilon} C C$ and satisfies a Lipschitz condition on every finite set and if $f_{0}$ is continuous, then the relation $f_{n}-v \rightarrow f_{0}$ implies $\phi: f_{n}-v \rightarrow \phi: f_{0}$.

That the statement is not true is illustrated by the following example. Let $f_{n}(t)=t+i / n$ for $0 \leqq t \leqq 1 ; n=1,2,3, \cdots$. Let $f_{0}(t)=t$. It is clear that $f_{n}-v \rightarrow f_{0}$. Define

$$
\begin{aligned}
u(x, y)=\frac{\sin n^{4} \pi x}{n^{4} \pi} & \\
& +\left\{\frac{\sin (n-1)^{4} \pi x}{(n-1)^{4} \pi}-\frac{\sin n^{4} \pi x}{n^{4} \pi}\right\} \sin ^{2}\left\{\frac{\pi n(n-1)}{2}\left(y-\frac{1}{n}\right)\right\},
\end{aligned}
$$

if $1 / n \leqq y<1 /(n-1)$, where $n \geqq 2$;

$$
u(x, y)=\frac{\sin \pi x}{\pi} \quad \text { if } \quad y \geqq 1 ; \quad u(x, y)=0 \quad \text { if } \quad y \leqq 0 ;
$$

and let $\phi(x+i y)=u(x, y)$ for $-\infty<x, y<\infty$. Since the first partial derivatives of $u$ exist everywhere and are dominated by 3 , it follows that $\phi$ satisfies a Lipschitz condition on the complex plane. But

$$
\left\|\phi: f_{n}\right\|=\int_{0}^{1}\left|\cos n^{4} \pi x\right| d x \geqq \int_{0}^{1} \cos ^{2} n^{4} \pi x d x=\frac{1}{2} \quad(n=1,2,3, \cdots)
$$

with $\left\|\phi: f_{0}\right\|=0$ so that it is not true that $\phi: f_{n}-v \rightarrow \phi: f_{0}$. As a rough appraisal of the generality of Theorems 4.1 and 4.2 it is interesting to note that a function in $C C$ may be applicable to $f_{n}$ for $n=0,1,2$, without satisfying a Lipschitz condition on every bounded set in the complex plane.

5. Convergence in length. As our first application of preceding results we have the following theorem, which is a result obtained in a different way in AL.

THeOREM 5.1. The relation $Y_{n}-l \rightarrow Y_{0}$ implies $Y_{n}-v \rightarrow Y_{0}$.

Let $\phi(x+i y)=y,(-\infty<x, y<\infty)$. Now $\phi$ is strictly applicable to $I+i Y_{n}$. for $n=0,1,2, \cdots$ and since $\left(I+i Y_{n}\right)-v \rightarrow\left(I+i Y_{0}\right)$ we conclude that $\phi:\left(I+i Y_{n}\right)-v \rightarrow \phi:\left(I+i Y_{0}\right)$. Hence the theorem is established.

Corollary 5.1. The relation $Y_{n}-u l \rightarrow Y_{0}$ implies $Y_{n}-u v \rightarrow Y_{0}$.

We introduce here the notion of a singular function. 
Definition 5.1. If $f$ is of b.v. on $[a, b]$ with $f^{\prime}(t)=0$ for almost all $t$ on $[a, b]$ then $f$ is said to be singular on $[a, b]$.

A well known property of singular functions is this: Let $f$ be a singular function in $B V$ and assume $g$ an a.c. function in $B V$. Then

$$
T_{0}^{1}(f+g)=T_{0}^{1}(f)+T_{0}^{1}(g),
$$

so that if either $f(0)=0$ or $g(0)=0$, it is clear that $\|f+g\|=\|f\|+\|g\|$. We are now prepared to prove the following

THEOREM 5.2. If $Y_{0}$ is a singular function in $R B V$, then the relations $Y_{n}-l \rightarrow Y_{0}$ and $Y_{n}-v \rightarrow Y_{0}$ are equivalent.

Supposing $Y_{n}-v \rightarrow Y_{0}$ we deduce the relation

$$
\begin{aligned}
\left\|I+i Y_{0}\right\| & \leqq \liminf _{n \rightarrow \infty}\left\|I+i Y_{n}\right\| \leqq \limsup _{n \rightarrow \infty}\left\|I+i Y_{n}\right\| \leqq \limsup _{n \rightarrow \infty}\left\|Y_{n}\right\|+\|I\| \\
& =\|I\|+\left\|Y_{0}\right\|=\|I\|+\left\|i Y_{0}\right\|=\left\|I+i Y_{0}\right\|,
\end{aligned}
$$

which proves $Y_{n}-l \rightarrow Y_{0}$.

Application of Theorem 5.1 completes the proof.

TheOREM 5.3. If $u$ is applicable $(R)$ to $Y_{n}$ for $n=0,1,2, \cdots$ and in addition if $u$ is strictly applicable $(R)$ to $Y_{0}$, then the relation $Y_{n}-l \rightarrow Y_{0}$ implies

$$
\left(u \mid Y_{n}\right)-l \rightarrow\left(u \mid Y_{0}\right) \text {. }
$$

Define $\phi(x+i y)=x+i u(x, y)$ for $-\infty<x, y<\infty$. Now $\phi$ is applicable to $\left(I+i Y_{n}\right)$ for $n=0,1,2, \cdots$. It is also strictly applicable to $\left(I+i Y_{0}\right)$. Hence $\phi:\left(I+i Y_{n}\right)-v \rightarrow \phi:\left(I+i Y_{0}\right)$ or $\left\{I+i\left(u \mid Y_{n}\right)\right\}-v \rightarrow\left\{I+i\left(u \mid Y_{0}\right)\right\}$.

THEOREM 5.4. If $X_{0}$ is a.c., then the relations $X_{n}-l \rightarrow X_{0}$ and $Y_{n}-l \rightarrow Y_{0}$ imply the relations $\dagger$

$$
\begin{aligned}
\left(X_{n}+Y_{n}\right)-l & \rightarrow\left(X_{0}+Y_{0}\right), \\
X_{n} Y_{n}-l & \rightarrow X_{0} Y_{0} .
\end{aligned}
$$

Let $\Psi$ be a.c. on $\underset{x}{E}[-\infty<x<\infty]$ with $\Psi(x)=X_{0}(x)$ for $x$ on $[0,1]$. Define $u_{1}(x, y)=y-\Psi(x), u_{2}(x, y)=y+\Psi(x) ;-\infty<x, y<\infty$. Thus by Theorem 5.3 we have $\left(u_{1} \mid X_{n}\right)-l \rightarrow \theta$, which implies (Theorem 5.2) that $\left\|X_{n}-X_{0}\right\| \rightarrow 0$ (see $\$ 2$ for definition of $\theta$ ). Hence

$$
\left\|I+i\left(Y_{n}+X_{n}-X_{0}\right)\right\| \leqq\left\|I+i Y_{n}\right\|+\left\|X_{n}-X_{0}\right\| \rightarrow\left\|I+i Y_{0}\right\|,
$$

whence, by using the semi-continuity property of total variation, we deduce

$$
\left(Y_{n}+X_{n}-X_{0}\right)-l \rightarrow Y_{0} .
$$

$\dagger$ This is a generalization of Theorem 6 in AL.

If the assumption that $X_{0}$ is a.c. is deleted the theorem ceases to be true. See $\mathrm{AL}$, page 23 . 
This gives, in view of Theorem 5.3,

$$
\left[u_{2} \mid\left(Y_{n}+X_{n}-X_{0}\right)\right]-l \rightarrow\left(u_{2} \mid Y_{0}\right) \text { or }\left(Y_{n}+X_{n}\right)-l \rightarrow\left(Y_{0}+X_{0}\right) .
$$

Now by Lemma 3.2 we have

$$
\left\|Y_{n} \cdot\left(X_{n}-X_{0}\right)\right\| \leqq\left\|Y_{n}\right\| \cdot\left\|X_{n}-X_{0}\right\| \rightarrow 0,
$$

so that upon defining $u_{3}(x, y)=y \Psi(x),-\infty<x, y<\infty$, it is seen by Theorem 5.3 that

$$
\left(u_{3} \mid Y_{n}\right)-l \rightarrow\left(u_{3} \mid Y_{0}\right) \quad \text { or } \quad X_{0} Y_{n}-l \rightarrow X_{0} Y_{0}
$$

and since (Theorem 5.2)

$$
\left(X_{n} Y_{n}-X_{0} Y_{n}\right)=\left[Y_{n} \cdot\left(X_{n}-X_{0}\right)\right]-l \rightarrow \theta,
$$

we conclude upon adding, $\theta$ being a.c., that $X_{n} Y_{n}-l \rightarrow X_{0} Y_{0}$.

Lemma 5.1. Let $X$ be an a.c. point in the space $R B V$. The relation $\left(c I+Y_{n}\right)-v \rightarrow\left(c I+Y_{0}\right)$ for all real c implies $\left(X+Y_{n}\right)-v \rightarrow\left(X+Y_{0}\right)$.

Let $[a, b]$ be a subinterval of $[0,1]$. From Lemma 3.1 it follows that

$$
T_{t=a}^{b}\left(c_{1}+c_{2} t+Y_{n}(t)\right) \rightarrow T_{t=a}^{b}\left(c_{1}+c_{2} t+Y_{0}(t)\right)
$$

for all real $c_{1}$ and $c_{2}$, whence we conclude

$$
\left(\beta+Y_{n}\right)-v \rightarrow\left(\beta+Y_{0}\right),
$$

where $\beta$ is any polygonal function in $R B V$.

Let $\left\{\beta_{p}\right\}$ be a sequence of polygonal functions in $R B V$ such that as $p \rightarrow \infty$ $\beta_{p}-l \rightarrow X$. From Theorems 5.4 and 5.1 follow the relations $\left\|\beta_{p}-X\right\| \rightarrow 0$ and $\left(\beta_{p}+Y_{0}\right)-v \rightarrow\left(X+Y_{0}\right)$ as $p \rightarrow \infty$. Hence

$$
\begin{aligned}
\left\|X+Y_{0}\right\| & \leqq \liminf _{n \rightarrow \infty}\left\|X+Y_{n}\right\| \leqq \limsup _{n \rightarrow \infty}\left\|X+Y_{n}\right\| \\
& \leqq \limsup _{n \rightarrow \infty}\left\|\beta_{p}+Y_{n}\right\|+\lim _{n \rightarrow \infty}\left\|X-\beta_{p}\right\| \\
& =\left\|\beta_{p}+Y_{0}\right\|+\left\|X-\beta_{p}\right\| \rightarrow\left\|X+Y_{0}\right\| \text { as } p \rightarrow \infty
\end{aligned}
$$

and the lemma is proved.

THEOREM 5.5. The relation $\dagger\left(c I+Y_{n}\right)-v \rightarrow\left(c I+Y_{0}\right)$ for all real numbers $c$ and the relation $Y_{n}-l \rightarrow Y_{0}$ are equivalent.

Let $\alpha(t)=\int_{0}^{t} Y_{0}^{\prime}(s) d s$ and $\beta(t)=Y_{0}(t)-\alpha(t)$ for $t$ on $[0,1]$. From the preceding lemma follows

† We are indebted to Professor E. J. McShane for raising the question as to whether the relation $\left(c I+Y_{n}\right)-v \rightarrow\left(c I+Y_{0}\right)$ for all real numbers $c$ implies $Y_{n}-l \rightarrow Y_{0}$. 


$$
\left(Y_{n}-\alpha\right)-v \rightarrow \beta .
$$

Since $\beta$ is singular we deduce from Theorems 5.2 and 5.4 that

$$
\left(Y_{n}-\alpha\right)-l \rightarrow \beta \text { and } Y_{n}-l \rightarrow(\alpha+\beta)=Y_{0} .
$$

From Theorem 5.4 the converse follows immediately.

If $Y_{0}$ is a.c. then it appears, as a consequence of Theorems 5.1 and 5.4, that the relation $Y_{n}-l \rightarrow Y_{0}$ implies

$$
\int_{0}^{1}\left|Y_{n}^{\prime}(t)-Y_{0}^{\prime}(t)\right| d t \rightarrow 0 .
$$

However, if $Y_{0}$ is not a.c., this conclusion need not be true. It is true, nevertheless, that $Y_{n}^{\prime}$ converges to $Y_{0}^{\prime}$ in a manner intermediate between convergence in the mean and convergence in measure. To characterize this type of convergence we introduce the following definitions which may have some intrinsic interest.

Definition 5.2. If $f_{n}$ is measurable on a set $E$ for $n=1,2,3, \ldots$ and if corresponding to every $\epsilon>0$ there exists a measurable set $E_{1} \subset E$ of measure $>|E|-\epsilon$ such that

$$
\lim _{m, n \rightarrow \infty} \int_{E_{1}}\left|f_{m}(t)-f_{n}(t)\right| d t=0,
$$

then $\left\{f_{n}\right\}$ is said to be convergent almost in the mean on $E$.

DefFinition 5.3. If $f_{n}$ is measurable on a set $E$ for $n=0,1,2, \ldots$ and if corresponding to every $\epsilon>0$ there exists a measurable set $E_{1} \subset E$ of measure $>|E|-\epsilon$ such that

$$
\int_{E_{1}}\left|f_{n}(t)-f_{0}(t)\right| d t \rightarrow 0,
$$

then $f_{n}$ is said to converge almost in the mean to $f_{0}$ on $E$.

If $\left\{f_{n}\right\}$ is convergent almost in the mean on $E$ then it is easily seen that there exists a function $f_{0}$ defined on $E$ such that $f_{n}$ converges almost in the mean to $f_{0}$ on $E$.

Definition 5.4. By $f_{n}-\mu \rightarrow f_{0}$ is meant this: $f_{n}$ is in $C R$ for $n=0,1,2, \ldots$ and $f_{n}$ converges almost in the mean to $f_{0}$ on $[0,1]$.

THEOREM 5.6. The relation $Y_{n}-l \rightarrow Y_{0}$ implies and is implied by the two relations $Y_{n}-v \rightarrow Y_{0}$ and $Y_{n}^{\prime}-\mu \rightarrow Y_{0}^{\prime}$.

We have already seen (Theorem 5.1) that $Y_{n}-l \rightarrow Y_{0}$ implies $Y_{n}-v \rightarrow Y_{0}$. We now propose to show that $Y_{n}-l \rightarrow Y_{0}$ implies $Y_{n}^{\prime}-\mu \rightarrow Y_{0}^{\prime}$.

Define 


$$
\alpha(t)=\int_{0}^{t} Y_{0}(s) d s, \quad \beta(t)=Y_{0}(t)-\alpha(t) \text { for } t \text { on }[0,1],
$$

and let $\epsilon$ be any positive number. The singularity of $\beta$ implies, as is well known, the existence for each $m=1,2,3, \cdots$ of non-overlapping intervals $\left[a_{m, 1}, b_{m, 1}\right],\left[a_{m, 2}, b_{m, 2}\right], \cdots,\left[a_{m, N_{m}}, b_{m, N_{m}}\right]$ contained in $[0,1]$ such that

$$
\sum_{j=1}^{N_{m}} T_{a_{m, j}}^{b_{m, j}}(\beta)<\frac{1}{m}, \quad\left|A_{m}\right|>1-\frac{\epsilon}{2^{m}},
$$

where $A_{m}=\sum_{j=1}^{N m}\left[a_{m, j}, b_{m, j}\right]$. The absolute continuity of the function $\alpha$ implies that $\left(Y_{n}-\alpha\right)-l \rightarrow \beta$ which in turn implies $\left(Y_{n}-\alpha\right)-v \rightarrow \beta$. Letting $A=A_{1} A_{2} A_{3} \ldots$ we conclude from Lemmas 3.1 and 3.7 that

$$
\begin{aligned}
& \limsup \int_{n \rightarrow \infty}\left|Y_{n}^{\prime}(t)-Y_{0}^{\prime}(t)\right| d t=\limsup _{n \rightarrow \infty} \int_{A}\left|Y_{n}^{\prime}(t)-\alpha^{\prime}(t)\right| d t \\
& \leqq \limsup _{n \rightarrow \infty} \int_{A_{m}}\left|Y_{n}^{\prime}(t)-\alpha^{\prime}(t)\right| d t=\limsup _{n \rightarrow \infty} \sum_{j=1}^{N_{m}} \int_{a_{m, j}}^{b_{m, j}}\left|Y_{n}^{\prime}(t)-\alpha^{\prime}(t)\right| d t \\
& \leqq \limsup \sum_{j=1}^{N_{m}} T_{a_{m, j}}^{b_{m, j}}\left(Y_{n}-\alpha\right)=\sum_{j=1}^{N_{m}} T_{a_{m, j}}^{b_{m, j}}(\beta)<\frac{1}{m} \quad(m=1,2,3, \cdots)
\end{aligned}
$$

which implies $\int_{A}\left|Y_{n}^{\prime}(t)-Y_{0}^{\prime}(t)\right| d t \rightarrow 0$. Clearly $|A|>1-\epsilon$ so that from Definition 5.4 follows the relation $Y_{n}^{\prime}-\mu \rightarrow Y_{0}^{\prime}$.

Let us assume now that $Y_{n}-v \rightarrow Y_{0}$ and $Y_{n}^{\prime}-\mu \rightarrow Y_{0}^{\prime}$. Define $\alpha_{n}(t)=\int_{0}^{t} Y_{n}(s) d s, \quad \beta_{n}(t)=Y_{n}(t)-\alpha_{n}(t) \quad$ for $0 \leqq t \leqq 1 ; n=0,1,2, \cdots$, and let $\epsilon>0$. There exists a set $E \subset[0,1]$ such that $|E|>1-\epsilon$ and

$$
\int_{E}\left|\alpha_{n}^{\prime}(t)-\alpha_{0}^{\prime}(t)\right| d t=\int_{E}\left|Y_{n}^{\prime}(t)-Y_{0}^{\prime}(t)\right| d t \rightarrow 0 .
$$

Denoting by $E^{\prime}$ the complement of $E$ with respect to $[0,1]$ it is seen that the last relation combines with

to yield

$$
\begin{aligned}
\int_{E}\left|\alpha_{n}^{\prime}(t)\right| d t & +\int_{E^{\prime}}\left|\alpha_{n}^{\prime}(t)\right| d t+T_{0}^{1}\left(\beta_{n}\right)-\int_{E}\left|\alpha_{0}^{\prime}(t)\right| d t \\
& -\int_{E}\left|\alpha_{0}^{\prime}(t)\right| d t-T_{0}^{1}\left(\beta_{0}\right)=\left\{T_{0}^{1}\left(Y_{n}\right)-T_{0}^{1}\left(Y_{0}\right)\right\} \rightarrow 0
\end{aligned}
$$

$$
\left\{\int_{E}\left|\alpha_{n}^{\prime}(t)\right| d t+T_{0}^{1}\left(\beta_{n}\right)-\int_{E^{\prime}}\left|\alpha_{0}^{\prime}(t)\right| d t-T_{0}^{1}\left(\beta_{0}\right)\right\} \rightarrow 0 .
$$


Thus from the relation

$$
\begin{aligned}
T_{t=0}^{1} & \left\{t+i Y_{n}(t)\right\}-T_{t=0}^{1}\left\{t+i Y_{0}(t)\right\} \\
= & T_{t=0}^{1}\left\{t+i \alpha_{n}(t)\right\}+T_{0}^{1}\left(\beta_{n}\right)-T_{t=0}^{1}\left\{t+i \alpha_{0}(t)\right\}-T_{0}^{1}\left(\beta_{0}\right) \\
= & \int_{E}\left|1+i \alpha_{n}^{\prime}(t)\right| d t+\int_{E}\left|1+\alpha_{n}^{\prime}(t)\right| d t+T_{0}^{1}\left(\beta_{n}\right) \\
& -\int_{E}\left|1+i \alpha_{0}^{\prime}(t)\right| d t-\int_{E^{\prime}}\left|1+\alpha_{0}^{\prime}(t)\right| d t-T_{0}^{1}\left(\beta_{0}\right) \\
\leqq & \int_{E}\left|\alpha_{n}^{\prime}(t)-\alpha_{0}^{\prime}(t)\right| d t+\int_{E^{\prime}}\left|\alpha_{n}^{\prime}(t)\right| d t+T_{0}^{1}\left(\beta_{n}\right) \\
& -\int_{E^{\prime}}\left|\alpha_{0}^{\prime}(t)\right| d t-T_{0}^{1}\left(\beta_{0}\right)+2\left|E^{\prime}\right|,
\end{aligned}
$$

which holds for $n=1,2,3, \cdots$ we conclude

$$
\begin{aligned}
0 & \leqq \liminf _{n \rightarrow \infty} T_{t=0}^{1}\left\{t+i Y_{n}(t)\right\}-T_{t=0}^{1}\left\{t+i Y_{0}(t)\right\} \\
& \leqq \limsup _{n \rightarrow \infty} T_{t=0}^{1}\left\{t+i Y_{n}(t)\right\}-T_{t=0}^{1}\left\{t+i Y_{0}(t)\right\} \leqq 2\left|E^{\prime}\right|<2 \epsilon .
\end{aligned}
$$

The arbitrariness of $\epsilon$ completes the proof.

CoRollary 5.2. The relation $Y_{n}-l \rightarrow Y_{0}$ implies and is implied by the two relations $Y_{n}-v \rightarrow Y_{0}$ and $Y_{n}^{\prime}$ converges in measure to $Y_{0}^{\prime}$ on $[0,1]$.

Convergence in measure implies almost convergence in the mean of a subsequence.

An immediate consequence is

TheORem 5.7. Let $X_{n}-l \rightarrow X_{0}$ and $Y_{n}-l \rightarrow Y_{0}$. Then a necessary and sufficient condition for $\left(X_{n}+Y_{n}\right)-l \rightarrow\left(X_{0}+Y_{0}\right)$ is that $\left(X_{n}+Y_{n}\right)-v \rightarrow\left(X_{0}+Y_{0}\right)$; furthermore, a necessary and sufficient condition for $X_{n} Y_{n}-l \rightarrow X_{0} Y_{0}$ is that $X_{n} Y_{n}-v \rightarrow X_{0} Y_{0}$.

TheOReM 5.8. Let $Y_{n}$ be in $R B V$ with $P_{n}(t)$ and $N_{n}(t)$ denoting the positive and negative variations of $Y_{n}$ on $[0,1],(n=0,1,2, \cdots ; 0 \leqq t \leqq 1)$. Then the relation $Y_{n}-l \rightarrow Y_{0}$ implies the relations $P_{n}-l \rightarrow P_{0}$ and $N_{n}-l \rightarrow N_{0}$.

To prove this theorem verify first the relations $\dagger P_{n}-v \rightarrow P_{0}, N_{n}-v \rightarrow N_{0}$, and

$$
\frac{1}{2}\left(P_{n}-N_{n}\right)-l \rightarrow \frac{1}{2}\left(P_{0}-N_{0}\right), \quad \frac{1}{2}\left(P_{n}+N_{n}\right)-l \rightarrow \frac{1}{2}\left(P_{0}+N_{0}\right) .
$$

The desired conclusions are now immediate consequences of Theorem 5.7.

† AC, Theorem 1; AL, Corollary to Theorem 1. 
6. Uniform convergence in length. Theorem 4.2 combined with the methods used in proving Theorem 5.3 yields

Theorem 6.1. If $u$ is applicable $(R)$ to $Y_{n}$ for $n=0,1,2, \cdots$, then the relation $Y_{n}-u l \rightarrow Y_{0}$ implies

$$
\left(u \mid Y_{n}\right)-u l \rightarrow\left(u \mid Y_{0}\right) .
$$

We now recall the concept of an elementary step-function and of a singular function of the saltus type.

Definition 6.1. A function $f$ defined on $[a, b]$ is said to be an elementary step-function there if there exists a real number $c$ on $[a, b]$ and complex numbers $\gamma_{1}, \gamma_{2}, \gamma_{3}$, such that

$$
f(t)=\gamma_{1} \text {, if } 0 \leqq t<c ; f(c)=\gamma_{2} ; f(t)=\gamma_{3} \text { if } c<t \leqq b .
$$

Definition 6.2. A function $f$ of b.v. on $[a, b]$ is said to be a singular function of the saltus type on $[a, b]$ if there exist elementary step-functions $f_{1}, f_{2}, f_{3}, \cdots$ defined on $[a, b]$ such that

$$
f(t)=\sum_{n=1}^{\infty} f_{n}(t), \quad a \leqq t \leqq b ; \quad T_{a}^{b}(f)=\sum_{n=1}^{\infty} T_{a}^{b}\left(f_{n}\right) .
$$

It is readily seen that a singular function of the saltus type is singular, though we shall not make explicit use of this property. From the definition of pseudo-absolute continuity it follows that if $f$ is a pseudo-absolutely continuous function in $B V$, then there exists an a.c. function $\alpha$ in $B V$ and a singular function $\beta$ of the saltus type in $B V$ such that $f=\alpha+\beta$.

LEMma 6.1. If $\beta$ is an elementary step-function in $B V$, then the relation $f_{n}-u v \rightarrow f_{0}$ implies the relation

$$
\left(f_{n}+\beta\right)-u v \rightarrow\left(f_{0}+\beta\right) .
$$

There exist a real number $c$ on $[0,1]$ and complex numbers $\gamma_{1}, \gamma_{2}, \gamma_{3}$, such that $\beta(t)=\gamma_{1}$ if $0 \leqq t<c, \beta(c)=\gamma_{2}$, and $\beta(t)=\gamma_{3}$ if $c<t \leqq 1$, so that as a consequence of Lemma 3.1 it may be deduced that

$$
\begin{aligned}
& T_{0}^{c-}\left(f_{n}+\beta\right)=T_{t=0}^{c-}\left\{f_{n}(t)+\gamma_{1}\right\}=T_{0}^{c-}\left(f_{n}\right) \rightarrow T_{0}^{c-}\left(f_{0}\right) \\
& =T_{t=0}^{c-}\left\{f_{0}(t)+\gamma_{1}\right\}=T_{0}^{c}\left(f_{0}+\beta\right), \\
& \left|f_{n}(c)+\gamma_{2}-f_{n}(c-)-\gamma_{1}\right|+\left|f_{n}(c+)+\gamma_{3}-f_{n}(c)-\gamma_{2}\right| \\
& \rightarrow\left|f_{0}(c)+\gamma_{2}-f_{0}(c-)-\gamma_{1}\right|+\left|f_{0}(c+)+\gamma_{3}-f_{0}(c)-\gamma_{2}\right|, \\
& \begin{aligned}
T_{c+}^{1}\left(f_{n}+\beta\right) & =T_{t=c+}^{1}\left\{f_{n}(t)+\gamma_{3}\right\}=T_{c+}^{1}\left(f_{n}\right) \rightarrow T_{c+}^{1}\left(f_{0}\right) \\
& =T_{t=c+}^{1}\left\{f_{0}(t)+\gamma_{3}\right\}=T_{c+}^{1}\left(f_{0}+\beta\right) .
\end{aligned}
\end{aligned}
$$

Combining these three relations establishes the lemma. 
LEMma 6.2. If $\beta$ (in $B V$ ) is a singular function of the saltus type, then the relation $f_{n}-u v \rightarrow f_{0}$ implies the relation $\left(f_{n}+\beta\right)-u v \rightarrow\left(f_{0}+\beta\right)$.

There exist elementary step-functions $\beta_{1}, \beta_{2}, \beta_{3}, \cdots$ such that $\beta(t)$ $=\sum_{j=1}^{\infty} \beta_{j}(t)$ for $t$ on $[0,1]$ with $T_{0}{ }^{1}(\beta)=\sum_{j=1}^{\infty} T_{0}{ }^{1}\left(\beta_{j}\right)$. Hence $T_{0}{ }^{1}\left(B_{p}-\beta\right) \rightarrow 0$, where $B_{p}(t)=\sum_{j=1}^{p} \beta_{j}(t)$. From the preceding lemma we conclude (by induction) that

$$
\lim _{n \rightarrow \infty}\left\|f_{n}+B_{p}\right\|=\left\|f_{0}+B_{p}\right\| \quad(p=1,2,3, \cdots) .
$$

Thus follows

$$
\begin{aligned}
\left\|f_{0}+\beta\right\| & \leqq \liminf _{n \rightarrow \infty}\left\|f_{n}+\beta\right\| \leqq \limsup _{n \rightarrow \infty}\left\|f_{n}+\beta\right\| \\
& \leqq \limsup _{n \rightarrow \infty}\left\|f_{n}+B_{p}\right\|+\left\|\beta-B_{p}\right\|=\left\|f_{0}+B_{p}\right\|+\left\|\beta-B_{p}\right\| \\
& \leqq\left\|f_{0}+\beta\right\|+2\left\|\beta-B_{p}\right\| \rightarrow\left\|f_{0}+\beta\right\| \text { as } p \rightarrow \infty,
\end{aligned}
$$

and the proof is completed.

LEMMA 6.3. If $X_{0}$ is an a.c. function in $R B V$, then the relations $X_{n}-l \rightarrow X_{0}$ and $Y_{n}-l \rightarrow Y_{0}$ imply the relation $\left(X_{n}+i Y_{n}\right)-v \rightarrow\left(X_{0}+i Y_{0}\right)$.

Define $\alpha(t)=Y_{0}(0)+\int_{0}^{t} Y^{\prime}(s) d s, \beta(t)=Y(t)-\alpha(t)$ for $t$ on [0,1] noting that $\beta$ is singular with $\beta(0)=0$. From Theorems 5.1 and 5.4 we have $\left\|Y_{n}-\alpha\right\| \rightarrow\|\beta\|$ and since $\left\|X_{n}-X_{0}\right\| \rightarrow 0$ we deduce $\left\|X_{n}+i \alpha\right\| \rightarrow\left\|X_{0}+i \alpha\right\|$, so that

$$
\begin{aligned}
\left\|X_{n}+i Y_{n}\right\| & =\left\|X_{n}+i\left(Y_{n}-\alpha+\alpha\right)\right\| \\
& \leqq\left\|X_{n}+i \alpha\right\|+\left\|i\left(Y_{n}-\alpha\right)\right\| \rightarrow\left\|X_{0}+i \alpha\right\|+\|i \beta\| \\
& =\left\|X_{0}+i(\alpha+\beta)\right\|=\left\|X_{0}+i Y_{0}\right\|,
\end{aligned}
$$

and the proof is complete (by semi-continuity).

THEOREM 6.2. If $X_{0}$ is a pseudo-absolutely continuous function in $R B V$, then the relations $X_{n}-u l \rightarrow X_{0}$ and $Y_{n}-u l \rightarrow Y_{0}$ imply the relations

$$
\left(X_{n}+Y_{n}\right)-u l \rightarrow\left(X_{0}+Y_{0}\right) \text { and } X_{n} Y_{n}-u l \rightarrow X_{0} Y_{0} .
$$

Since $X_{0}$ is pseudo-absolutely continuous we conclude the existence of an a.c. function $\alpha$ in $R B V$ and a singular function $\beta$ of the saltus type likewise in $R B V$ for which $X_{0}=\alpha+\beta$. As a corollary of Lemma $6.2,\left(X_{n}-\beta\right)-u l \rightarrow \alpha$, and since $Y_{n}-u l \rightarrow Y_{0}$ it is seen from Lemma 6.3 that

$$
\left(X_{n}-\beta+i Y_{n}\right)-u v \rightarrow\left(\alpha+i Y_{0}\right),
$$

so that using Lemma 6.2 again, yields

$$
\left(X_{n}+i Y_{n}\right)-u v \rightarrow\left(\alpha+\beta+i Y_{0}\right)=\left(X_{0}+i Y_{0}\right) .
$$


Letting $\phi_{1}(x+i y)=x+y$ and $\phi_{2}(x+i y)=x y$ for $-\infty<x, y<\infty$ we conclude from Theorem 4.2 that

$$
\begin{aligned}
& \phi_{1}:\left(X_{n}+i Y_{n}\right)-u v \rightarrow \phi_{1}:\left(X_{0}+i Y_{0}\right), \\
& \phi_{2}:\left(X_{n}+i Y_{n}\right)-u v \rightarrow \phi_{2}:\left(X_{0}+i Y_{0}\right) .
\end{aligned}
$$

That is,

$$
\left(X_{n}+Y_{n}\right)-u v \rightarrow\left(X_{0}+Y_{0}\right) \text { and } X_{n} Y_{n}-u v \rightarrow X_{0} Y_{0} .
$$

Application of Theorem 5.7 completes the proof.

7. Strong convergence. It is at once apparent that strong convergence implies every other type considered in this paper, and also that it is invariant under addition and multiplication. It is natural to ask if Theorem 6.1 likewise holds for strong convergence. The answer is yes, but before proving this we state as an obvious corollary of Lemma 4.5 the following

LEMMA 7.1. If $Y$ is a pseudo-absolutely continuous function in $R B V$ and $u$ is applicable $(R)$ to $Y$, then $(u \mid Y)$ is likewise pseudo-absolutely continuous.

We are now prepared to prove

Theorem 7.1. If $u$ is applicable $(R)$ to $Y_{n}$ for $n=0,1,2, \cdots$, then the relation $Y_{n}-s \rightarrow Y_{0}$ implies

$$
\left(u \mid Y_{n}\right)-s \rightarrow\left(u \mid Y_{0}\right) .
$$

Since $Y_{0}$ is in $R B V$ there exist a continuous function $\alpha$ in $R B V$ and a singular function $\beta$ of the saltus type likewise in $R B V$ for which $Y_{0}=\alpha+\beta$. Defining

$$
S(t)=\frac{t+T_{0}^{t}(\alpha)}{1+T_{0}^{1}(\alpha)}
$$

it is seen that $S$ is a continuous increasing function in $R B V$. Clearly there exists an increasing function $\Psi$ satisfying a Lipschitz condition on $\underset{x}{E}[-\infty<x<\infty]$ for which $\Psi\{S(t)\}=t, 0 \leqq t \leqq 1$. Let $A(s)=\alpha\{\Psi(s)\}, B(s) \stackrel{x}{=} \beta\{\Psi(s)\}, \eta_{n}(s)$ $=Y_{n}\{\Psi(s)\},(0 \leqq s \leqq 1 ; n=0,1,2, \cdots)$. Finally let $u_{1}(x, y)=u(\Psi(x), y)$, $-\infty<x, y<\infty$.

First notice that

$$
\begin{aligned}
\left|A\left(s_{2}\right)-A\left(s_{1}\right)\right| & \leqq T_{\Psi\left(s_{1}\right)}^{\Psi(\alpha)}(\alpha) \Psi\left(s_{2}\right)-\Psi\left(s_{1}\right)+T_{\Psi\left(s_{1}\right)}^{\Psi(\alpha)}(\alpha) \\
& =\left(1+T_{0}^{1}(\alpha)\right)\left(S\left\{\Psi\left(s_{2}\right)\right\}-S\left\{\Psi\left(s_{1}\right)\right\}\right)=\left(1+T_{0}^{1}(\alpha)\right)\left(s_{2}-s_{1}\right)
\end{aligned}
$$

if $0 \leqq s_{1} \leqq s_{2} \leqq 1$, which implies absolute continuity of $A$. Since $\beta$ is a singular function of the saltus type, it may be seen from Lemma 3.8 that $B$ is likewise 
a singular function of the saltus type so that noting $\eta_{0}=A+B$ establishes the pseudo-absolute continuity of $\eta_{0}$. Now from the definitions involved and the fact that $\Psi$ is increasing and absolutely continuous it becomes apparent that if $u$ is $\Omega$, then $u_{1}$ is $\Omega$; if $u$ is $\Omega_{2}$, then $u_{1}$ is $\Omega_{2}$; and since monotonicity of $Y_{n}$ implies monotonicity of $\eta_{n}$ for $n=0,1,2, \cdots$, we conclude that $u_{1}$ is applicable $(R)$ to $\eta_{n}$ for $n=0,1,2, \cdots$. Another application of Lemma 3.8 yields the relation

$$
\left\|\eta_{n}-\eta_{0}\right\|=\left\|Y_{n}-Y_{0}\right\| \rightarrow 0,
$$

so that, since strong convergence implies uniform convergence in length, we may deduce successively (with the help of Lemma 7.1, Theorems 6.1 and 6.2) the following relations,

$$
\begin{gathered}
\eta_{n}-u l \rightarrow \eta_{0}, \quad\left(u_{1} \mid \eta_{n}\right)-u l \rightarrow\left(u_{1} \mid \eta_{0}\right), \quad\left\{\left(u_{1} \mid \eta_{n}\right)-\left(u_{1} \mid \eta_{0}\right)\right\}-u l \rightarrow \theta, \\
\left\|\left(u_{1} \mid \eta_{n}\right)-\left(u_{1} \mid \eta_{0}\right)\right\| \rightarrow 0 .
\end{gathered}
$$

Thus (Lemma 3.8)

$$
\begin{aligned}
& T_{t=0}^{1}\left\{u\left(t, Y_{n}(t)\right)-u\left(t, Y_{0}(t)\right)\right\}=T_{s=0}^{1}\left\{u\left[\Psi(s), Y_{n}(\Psi(s))\right]-u\left[\Psi(s), Y_{0}(\Psi(s))\right]\right\} \\
& \quad=T_{s=0}^{1}\left\{u\left(\Psi(s), \eta_{n}(s)\right)-u\left(\Psi(s), \eta_{0}(s)\right)\right\}=T_{s=0}^{1}\left\{u_{1}\left(s, \eta_{n}(s)\right)-u_{1}\left(s, \eta_{0}(s)\right)\right\} \\
& \quad \leqq\left\|\left(u_{1} \mid \eta_{n}\right)-\left(u_{1} \mid \eta_{0}\right)\right\| \rightarrow 0,
\end{aligned}
$$

which implies immediately

$$
\left\|\left(u \mid Y_{n}\right)-\left(u \mid Y_{0}\right)\right\| \rightarrow 0
$$

as was to be proved.

THEOREM 7.2. If $f_{0}$ is an a.c. function in $B V$, then a necessary and sufficient condition that $f_{n}-s \rightarrow f_{0}$ is that

$$
\left(c I+f_{n}\right)-v \rightarrow\left(c I+f_{0}\right),
$$

for all real numbers $c$.

The necessity being obvious we turn to the sufficiency. Let $X_{n}+i Y_{n}=f_{n}$ for $n=0,1,2, \cdots$, and define

$$
\phi_{1}(x+i y)=x, \phi_{2}(x+i y)=x+y \text {, for }-\infty<x, y<\infty .
$$

Since $\phi_{1}$ and $\phi_{2}$ are applicable to $\left(c I+f_{n}\right)$ for $n=0,1,2, \cdots$ and strictly applicable to $\left(c I+f_{0}\right)$ whatever real number $c$ may be, we conclude

$$
\left(c I+X_{n}\right)-v \rightarrow\left(c I+X_{0}\right),\left(c I+X_{n}+Y_{n}\right)-v \rightarrow\left(c I+X_{0}+Y_{0}\right)
$$

for all real numbers $c$. Whence, with the help of Theorems 5.5 and 5.4 follow successively the relations 


$$
\begin{gathered}
X_{n}-l \rightarrow X_{0}, \quad\left(X_{n}+Y_{n}\right)-l \rightarrow\left(X_{0}+Y_{0}\right), \quad Y_{n}-l \rightarrow Y_{0}, \\
\left\|X_{n}-X_{0}\right\| \rightarrow 0, \quad\left\|Y_{n}-Y_{0}\right\| \rightarrow 0, \quad\left\|f_{n}-f_{0}\right\| \rightarrow 0
\end{gathered}
$$

and the proof is complete.

8. Applications; generalizations of a theorem of Plessner and its converse. It is well known that if $p>1$ then the two relations

$$
\int_{0}^{t} f_{n}(s) d s \rightarrow \int_{0}^{t} f_{0}(s) d s
$$

and

$$
\int_{0}^{1}\left|f_{n}(t)\right|^{p} d t \rightarrow \int_{0}^{1}\left|f_{0}(t)\right|^{p} d t
$$

imply and are implied by the relation

$$
\int_{0}^{1}\left|f_{n}(t)-f_{0}(t)\right|^{p} d t \rightarrow 0 .
$$

It is likewise well known that the theorem is not true for $p=1$. The following theorem would therefore seem of some interest.

THE OREM 8.1. If $f_{n}$ is a summable function in $C R$ for $n=0,1,2, \cdots$, then the two relations

$$
\begin{aligned}
& \int_{0}^{t} f_{n}(s) d s \rightarrow \int_{0}^{t} f_{0}(s) d s \quad(0 \leqq t \leqq 1), \\
& \int_{0}^{1}\left|c+f_{n}(t)\right| d t \rightarrow \int_{0}^{1}\left|c+f_{0}(t)\right| d t \text { for all real numbers } c,
\end{aligned}
$$

imply and are implied by the relation

$$
\int_{0}^{1}\left|f_{n}(t)-f_{0}(t)\right| d t \rightarrow 0 .
$$

Obviously the last relation implies the first two. Assuming the first two relations to be true and defining $F_{n}(t)=\int_{0}^{t} f_{n}(s) d s$ (for $0 \leqq t \leqq 1$; $n=0,1,2, \cdots)$, we note from the first relation that $\left(c t+F_{n}(t)\right) \rightarrow\left(c t+F_{0}(t)\right)$ for $t$ on $[0,1]$, and from the second relation that

$$
T_{t=0}^{1}\left\{c t+F_{n}(t)\right\}=\int_{0}^{1}\left|c+f_{n}(t)\right| d t \rightarrow \int_{0}^{1}\left|c+f_{0}(t)\right| d t=T_{t=0}^{1}\left\{c t+F_{0}(t)\right\}
$$

for all real numbers $c$. Hence

$$
\left(c I+F_{n}\right)-v \rightarrow\left(c I+F_{0}\right) \text { for all real numbers } c,
$$


so that Theorem 7.2 implies

$$
\int_{0}^{1}\left|f_{n}(t)-f_{0}(t)\right| d t=T_{0}^{1}\left(F_{n}-F_{0}\right) \rightarrow 0
$$

which completes the proof.

A result of Ursell combined with a theorem of Plessner establishes the following theorem: $\dagger$

Let $f$ be a finite, real-valued, measurable function with period 1. If

then $f$ is a.c. on $[0,1]$.

$$
\lim _{h \rightarrow 0} T_{t \rightarrow 0}^{1}\{f(t+h)-f(t)\}=0,
$$

We now propose to generalize this theorem. First, however, it is convenient to prove the following

THEOREM 8.2. Let $f$ be a finite, real-valued, function with period 1 which is measurable on a set of positive measure. If there exists a non-vanishing function $g$ in $R B V$ such that

$$
\lim _{h \rightarrow 0} T_{t=0}^{1}\{f(t)+h g(t)\}=0,
$$

then $f$ is continuous on $[0,1]$.

Clearly there exists a closed set $D$ on $[0,1]$ of positive measure relative to which $f$ is continuous. Let $\beta$ be the characteristic function of this set and denote $\int_{0}^{v} \beta(x) d x$ by $B(y)$ for $-\infty<y<\infty$. Now $B$ satisfies a Lipschitz condition so that use of Theorems 7.1 and 3.1, Lemma 3.7, and Corollary 3.1 establishes the relation

$$
\begin{aligned}
0 & =\lim _{h \rightarrow 0} T_{t=0}^{1}\{B(t+h g(t))-B(t)\} \\
& \geqq \limsup _{h \rightarrow 0} \int_{0}^{1}\left|B^{\prime}(t+h g(t))\left(1+h g^{\prime}(t)\right)-B^{\prime}(t)\right| d t \\
& =\limsup _{h \rightarrow 0} \int_{0}^{1}\left|\beta(t+h g(t))\left(1+h g^{\prime}(t)\right)-\beta(t)\right| d t .
\end{aligned}
$$

Hence there exists a $\delta_{0}>0$ such that $|h|<\delta_{0}$ implies

$$
\int_{0}^{1}\left|\beta(t+h g(t))\left(1+h g^{\prime}(t)\right)-\beta(t)\right| d t<\int_{0}^{1}|\beta(t)| d t,
$$

† An elementary proof was given by N. Dunford, Bulletin of the American Mathematical Society, vol. 41 (1935), pp. 356-358. 
so that corresponding to each $h^{*}$ for which $\left|h^{*}\right|<\delta_{0}$ there exists a point $t^{*}$ in $D$, such that $t^{*}+h^{*} g\left(t^{*}\right)$ is likewise in $D$; for assuming the contrary leads immediately to a contradiction of the above relation.

Let $\epsilon$ be any positive number. Since $D$ is closed there exists a $\delta_{1}>0$ such that, $|h|<\delta_{1}$ implies

$$
|f(t+h g(t))-f(t)|<\frac{\epsilon}{2}
$$

for all $t$ on $[0,1]$ for which $t$ and $t+h g(t)$ are both in $D$. By hypothesis there exists a $\delta_{2}>0$ such that $|h|<\delta_{2}$ implies

$$
T_{t=0}^{1}\{f(t+h g(t))-f(t)\}<\frac{\epsilon}{2} .
$$

Let $\delta$ be the least of the numbers $\delta_{0}, \delta_{1}, \delta_{2}$, and let $h_{0}$ be any number $<\delta$ in absolute value. As we have seen, there exists a point $t_{0}$ in $D$ such that $t_{0}+h_{0} g\left(t_{0}\right)$ is likewise in $D$. Hence

$$
\begin{aligned}
\left|f\left(t_{1}+h_{0} g\left(t_{1}\right)\right)-f\left(t_{1}\right)\right| \leqq & \left|f\left(t_{1}+h_{0} g\left(t_{1}\right)\right)-f\left(t_{1}\right)-f\left(t_{0}+h_{0} g\left(t_{0}\right)\right)+f\left(t_{0}\right)\right| \\
& +\left|f\left(t_{0}+h_{0} g\left(t_{0}\right)\right)-f\left(t_{0}\right)\right| \\
< & T_{t=0}^{1}\left\{f\left(t+h_{0} g(t)\right)-f(t)\right\}+\frac{\epsilon}{2}<\frac{\epsilon}{2}+\frac{\epsilon}{2}=\epsilon
\end{aligned}
$$

for $t_{1}$ on $[0,1]$. Hence $f$ is continuous on $[0,1]$ and the proof is complete.

It should be noted that the only place in the proof where a result of this paper is used is in proving

$$
\lim _{h \rightarrow 0} \int_{0}^{1}\left|\beta(t+h g(t))\left(1+h g^{\prime}(t)\right)-\beta(t)\right| d t=0 .
$$

However if $g(t)=1$ for $t$ on $[0,1]$, this relation is an immediate consequence of a well known theorem of Lebesgue, which in connection with the method used in proving Theorem 8.3 leads to a proof of the Plessner theorem which is independent of the preceding results in this paper.

We now turn to

TheOREM 8.3. Let $f$ be a finite, real-valued function with period 1 which is measurable on a set of positive measure. If there exist a function $g$ in $R B V$ and a positive number $r$ such that

$$
\lim _{h \rightarrow 0} T_{t=0}^{1}\{f(t+h g(t))-f(t)\}=0
$$

with $|g(t)|>$ for ton $[0,1]$, then $f$ is a.c. on $[0,1]$. 
Let $\delta>0$ be such that $T_{t=0}^{1} U(t, h)<1$ for $|h| \leqq \delta$, where $U(t, h)$ $=[f(t+h g(t))-f(t)] g(t)$ for $0 \leqq t \leqq 1, h \leqq \delta$; let $\alpha(t)=1 / g(t),(0 \leqq t \leqq 1)$; let $r_{0}=r^{-1}$ and note that $\|\alpha\|<\infty,|\alpha(t)|<r_{0}$ for $t$ on $[0,1]$; let $V(h)=T_{t-0}^{1-0} U(t, h)$ for $|h| \leqq \delta$. Since $V$ is bounded on $[-\delta, \delta]$ and $f$ is continuous by the previous theorem, the semi-continuity property of total variation shows that $V$ is lower semi-continuous and hence summable on $[-\delta, \delta]$. Let

$$
M(h)=\sup _{0 \leqq t \leqq 1,|\varepsilon| \leqq h} U(t, s) \quad(0 \leqq h \leqq \delta)
$$

remarking that $M$ is monotone on $[0, \delta]$ and $M(h) \rightarrow 0$ as $h \rightarrow 0+$. Define

$$
F_{h}(t)=\frac{1}{h} \int_{t}^{t+h} f(s) d s \quad\left(0 \leqq t \leqq 1,0 \leqq h \leqq r_{1}\right),
$$

where $r_{1}=\delta r$, and let $S$ be any partition of $[0,1]$ with $S \equiv\left(0=t_{0}<t_{1}<t_{2}\right.$ $\left.<\cdots<t_{N}=1\right)$.

From the relation

$$
\begin{aligned}
F_{h}(t)-f(t) & =\frac{1}{h} \int_{0}^{h}\{f(t+s)-f(t)\} d s \\
& =\frac{1}{h} \int_{0}^{h \alpha(t)}\{f(t+s g(t))-f(t)\} g(t) d s \\
& =\frac{1}{h} \int_{0}^{h \alpha(t)} U(t, s) d s \quad\left(0 \leqq t \leqq 1,0 \leqq h \leqq r_{1}\right)
\end{aligned}
$$

and the relation

$$
\begin{aligned}
\sum_{j=1}^{N} \mid & \frac{1}{h} \int_{0}^{h \alpha\left(t_{j}\right)} U\left(t_{j}, s\right) d s-\frac{1}{h} \int_{0}^{h \alpha\left(t_{j-1}\right)} U\left(t_{j-1}, s\right) d s \mid \\
& =\sum_{j=1}^{N}\left|\frac{1}{h} \int_{0}^{h \alpha\left(t_{j}\right)} U\left(t_{j}, s\right)-U\left(t_{j-1}, s\right) d s+\frac{1}{h} \int_{h \alpha\left(t_{j-1}\right)}^{h \alpha\left(t_{j}\right)} U\left(t_{j-1}, s\right) d s\right| \\
& \leqq \sum_{j=1}^{N}\left\{\frac{1}{h} \int_{-h r_{0}}^{h r_{0}}\left|U\left(t_{j}, s\right)-U\left(t_{j-1}, s\right)\right| d s+\frac{1}{h}\left|\int_{h \alpha\left(t_{j-1}\right)}^{h \alpha\left(t_{j}\right)} M\left(h r_{0}\right) d s\right|\right\} \\
& \leqq \frac{1}{h} \int_{-h r_{0}}^{h r_{0}} \sum_{j=1}^{N}\left|U\left(t_{j}, s\right)-U\left(t_{j-1}, s\right)\right| d s+\sum_{j=1}^{N} M\left(h r_{0}\right)\left|\alpha\left(t_{j}\right)-\alpha\left(t_{j-1}\right)\right| \\
& \leqq \frac{1}{h} \int_{-h r_{0}}^{h r_{0}} V(s) d s+M\left(h r_{0}\right)\|\alpha\| \quad \quad\left(0 \leqq h \leqq r_{1}\right),
\end{aligned}
$$

we deduce (since $S$ was arbitrary) the relation

$$
T_{0}^{1}\left(F_{h}-f\right) \leqq \frac{1}{h} \int_{-h r_{0}}^{h r_{0}} V(s) d s+M\left(h r_{0}\right)\|\alpha\| \quad\left(0 \leqq h \leqq r_{1}\right) .
$$


Now, Lemma 3.2 yields the relation

$$
V(s) \leqq\left[|f(s g(0))-f(0)|+T_{t=0}^{1}\{f(t+s g(t))-f(t)\}\right]|| g|| \quad\left(|s| \leqq r_{1}\right),
$$

so that $V(s) \rightarrow 0$ as $s \rightarrow 0$. Hence

$$
\lim _{h \rightarrow 0+} T_{0}^{1}\left\{F_{h}-f\right\}=0,
$$

where $F_{h}$ is a.c. for $0 \leqq h \leqq r_{1}$ which implies, as is well known, the absolute continuity of $f$ on $[0,1]$. This completes the proof.

From the results in $\$ 7$ it is clear that a variety of theorems concerning the behavior of $T_{t=0}^{1}\{f(t+h g(t))-f(t)\}$ as $h \rightarrow 0$ (or as $h \rightarrow 0+, h \rightarrow 0-$ ) can be readily proved. Among these is one which can be proved directly without great difficulty, and which forms the necessity part of the next and concluding theorem. This theorem is a simultaneous extension of Plessner's theorem and its converse.

THEOREM 8.4. Let $f$ be a finite, real-valued function with period 1 which is measurable on set of positive measure. Let $g$ be a non-vanishing function in $R B V$ which satisfies a Lipschitz condition. Then a necessary and sufficient condition that $f$ be a.c. on $[0,1]$ is that

$$
\lim _{h \rightarrow 0} T_{t=0}^{1}\{f(t+h g(t))-f(t)\}=0 .
$$

Simply note that $t+h g(t)$ increases with $t$ for $h$ sufficiently small and apply Theorem 7.1.

BROWN UNIVERSITY,

Providence, R. I. 